\title{
« Deux attitudes face au monde » : La criminologie à l'épreuve des illégalismes financiers
}

"Two Attitudes towards the World ».Criminology in face of Financial Illegalisms

\section{Anthony Amicelle}

\section{OpenEdition}

12 Journals

\section{Édition électronique}

URL : http://journals.openedition.org/conflits/18890

DOI : $10.4000 /$ conflits. 18890

ISSN : $1777-5345$

Éditeur :

CCLS - Centre d'études sur les conflits lilberté et sécurité, L'Harmattan

\section{Édition imprimée}

Date de publication : 31 décembre 2014

Pagination : 65-98

ISBN : 978-2-343-05760-6

ISSN : 1157-996X

Référence électronique

Anthony Amicelle, « «Deux attitudes face au monde » : La criminologie à l'épreuve des illégalismes financiers », Cultures \& Conflits [En ligne], 94-95-96 | été-automne-hiver 2014, mis en ligne le 20 février 2016, consulté le 30 mars 2021. URL : http://journals.openedition.org/conflits/18890 ; DOI : https:// doi.org/10.4000/conflits. 18890 


\section{«Deux attitudes face au monde» La criminologie à l'épreuve des illégalismes financiers 1}

\section{Anthony AMICELLE}

Anthony Amicelle est professeur adjoint au sein de l'École de criminologie de l'Université de Montréal, rattaché au Centre international de criminologie comparée (CICC) et au Centre d'études et de recherches internationales (CÉRIUM).

«Du côté francophone, c'est Surveiller et punir qui a donc fait charnière. Michel Foucault était ici à l'époque, à Montréal, où il avait quelques amis. Il avait la paix pour écrire ce livre. Nous nous sommes rencontrés, nous sommes sortis deux ou trois fois, une fois avec ma femme, je pense, et une fois seuls, manger ici dans le quartier, bavardant. Et comme je suis un bavard incorrigible, je parlais un peu plus que lui. Je commentais ce qu'il écrivait et je faisais un tour d'horizon de la discipline. Après la deuxième ou troisième sortie, très gentiment, il a dit: "Écoutez, j'éprouve beaucoup de plaisir à vous écouter, à échanger avec vous, mais si vous permettez, on en restera là. Je suis ici pour terminer un livre. Mon temps est extrêmement limité et, surtout, je veux prouver une thèse”. Il m'a dit ça littéralement. "Et à vous écouter je ne terminerai jamais parce qu'il y a ceci, il y a cela, il y a autre chose ; moi, je n'ai pas le temps de courir tous ces lièvres, je n'ai pas le temps de vérifier tout cela”. Et je n'oublierai jamais ses derniers mots: "Je veux rester dans un état d'ignorance créatrice. Et avec vous dans les environs, je vois que ce n'est pas possible”. Je ne l'ai plus revu. Vous voyez, cela vous montre un peu les deux approches, les deux perspectives, deux attitudes face au monde. [...] Après il y a eu le schisme, le tremblement de terre des “nouvelles" criminologies inspirées par Michel Foucault 2 ».

1. L'auteur tient à remercier les évaluateurs de la revue Cultures $E$ Conflits ainsi que Frédéric Ocqueteau et Jean Bérard pour leurs précieux commentaires sur une version antérieure du présent article.

2. Fournier M., Entretiens avec Denis Szabo. Fondation et fondements de la criminologie, Montréal, Liber, 1998, pp. 107-109. 
Touvel arrivant à l'École de criminologie de l’Université de Montréal, la curiosité a été grande pour moi de mieux connaître la réception de certaines œuvres, telles que celle de Michel Foucault, au sein de mon département et, par extension, au sein de ma «discipline d'adoption». À cet égard, la lecture des mémoires de Denis Szabo, fondateur de l'École, a constitué une entrée stimulante pour appréhender l'un des rôles attribués aux travaux de Foucault. Ce dernier y est dépeint sous les traits d'un théoricien qui aurait rompu l'unité de la communion criminologique en contribuant notamment « à retirer le "tapis empirique" sous la discipline ${ }^{3}$ ".

Si Surveiller et punir ${ }^{4}$ a pu provoquer une forme de rupture dans les manières de faire de la criminologie, l'usage dans cet ouvrage du terme «illégalismes » en est certainement une des clés de compréhension sur le plan conceptuel. Introduit en 1971 puis mobilisé lors du cours au Collège de France de 1972-1973 5 avant d'être repris dans le livre « charnière » de 1975, ce concept visait à dépasser la terminologie au fondement de la criminologie, à commencer par les notions d'infraction et de délinquance, pour précisément rompre avec deux préjugés encore tenaces. D'un côté, Foucault remettait en cause « la fausse neutralité des catégories juridiques qui présentent "l'ordre" et "le désordre" comme des faits historiques stables et universels, comme des faits objectifs dépourvus de tout jugement de valeur ${ }^{6}$ ». De l'autre, il critiquait « la fausse neutralité des catégories criminologiques qui attribuent à des déterminants individuels internes l'origine des actes de transgression sociale. Le placage de concepts médicaux interprétatifs a masqué au XIXe siècle les fondements politiques et sociaux des pratiques transgressives et de leur répres$\sin ^{7} \%$.

La réflexion en termes d'illégalismes rompait aussi avec une vision exclusivement parasitaire de la criminalité qui viendrait menacer de l'extérieur une vie économique et politique délestée pour l'occasion de ses contradictions internes. Toujours vivace, cette vision est portée par le titulaire de la seule chaire de criminologie tant controversée en France, Alain Bauer : «Il existe une face noire de la mondialisation (aussi ancienne que la mondialisation ellemême) qui menace l'économie et la finance mondiales. Côté obscur d'un phénomène licite, elle a été accélérée et accentuée par l'effondrement de l'ordre bipolaire du monde depuis 1989-91 et par le désordre consécutif. Clairement criminelle ou terroriste, elle se diffuse avec une telle vivacité par les interstices du système qu'elle perturbe désormais son fonctionnement même 8 ». Contre

3. Ibid., p. 109.

4. Foucault M., Surveiller et punir. Naissance de la prison, Paris, Gallimard, 1975.

5. Foucault M., La société punitive. Cours au Collège de France, 1972-1973, Paris, Seuil/Gallimard, 2013.

6. Lascoumes P., «L'illégalisme, outil d'analyse », Sociétés E Représentations, 3, 1996, p. 79.

7. Ibid., p. 80.

8. Bauer A., «L'invention de la criminalité globale », Cabiers de la sécurité intérieure, 25, 2013, p. 85 . 
cette perspective réduisant systématiquement la « dimension économique et financière » de la criminalité à l'envers de l'ordre social, l'approche par les illégalismes invite à resituer ces arrangements pratiques avec la loi au sein $\mathrm{du}$ fonctionnement des différents pans de la la société. Loin d'être confinés à une position d'extériorité et à un "Milieu » parasitaire, les illégalismes économiques et financiers doivent aussi et surtout être appréhendés comme une composante historique du monde des affaires ; c'est-à-dire moins comme un élément dysfonctionnel que comme un mécanisme inhérent au fonctionnement de l'ordre existant.

Plus généralement chez Foucault, la notion d'illégalismes traduit l'ambition, ou plutôt la nécessité, d'analyser les rapports entre la diversité des pratiques de jeu (contournement, détournement ou violation explicite) avec les normes en place et la variété des réponses apportées à ces comportements socialement et/ou juridiquement définis comme transgressifs 9. Dit autrement, il s'agit de " penser relationellement la position sociale des auteurs [d'illégalismes], les modalités pratiques de leurs actes et les diverses formes de réactions qu'ils suscitent de la part des instances de régulation 10 ». La rupture est ici consommée avec les études entièrement focalisées sur le passage à l'acte transgressif, susceptibles de masquer cette économie des pratiques et faire perdre de vue les activités institutionnelles de (dis)qualification des comportements posés comme délinquants. Cette « conception relationnelle du monde social 11 » invite également à se démarquer des approches portant uniquement sur le domaine pénal et presque exclusivement sur les personnes condamnées à des peines d'emprisonnement. En tenant compte du répertoire d'actions disponibles pour chaque type d'illégalismes, l'attention est tournée vers les dynamiques sociales, institutionnelles et conjoncturelles permettant de comprendre les différences de traitement constatées en matière de déviance et de criminalité. Il s'agit in fine de dessiner les contours d'une économie générale des illégalismes propre à une société donnée marquée par des rapports de domination spécifiques.

L'objectif du présent article est de questionner à nouveaux frais la valeur heuristique du concept d'illégalismes pour analyser les conduites économiques et financières indisciplinées et leur contrôle. Pour ce faire, nous commencerons par replacer ce concept clé dans son espace de formulation au sein des écrits de Michel Foucault. Dans les sections suivantes, nous nous attacherons aux modalités de réappropriation de cet appareillage conceptuel sur les problématiques de transgressions économiques et financières. Loin d'avoir retiré le tapis empirique sous le pied des chercheurs, «l'une des périodes les

9. Foucault M., Surveiller et punir, op. cit. ; Lascoumes P., «L'illégalisme, outil d'analyse ", op. cit.

10. Spire A., "Pour une approche sociologique de la délinquance en col blanc ", Champ pénal/Penal field [En ligne], vol. 10, 2013, p. 12.

11. Lacombe D., «Les liaisons dangereuses : Foucault et la criminologie », Criminologie, 26-1, 1993, pp. 51-72. 
plus militantes de Foucault 12 » a accouché de propositions théoriques ayant fourni un cadre d'analyse à des enquêtes de terrain rigoureuses. En insistant plus longuement sur le travail réalisé par Pierre Lascoumes depuis la fin des années 1970 et sur les contributions récentes d'Alexis Spire, il s'agira d'expliciter les façons dont ce programme de recherche foucaldien a pu et peut encore être réinvesti et enrichi au gré de nouveaux intérêts de connaissance et surtout de nouvelles configurations sociales. À ce titre, le but n'est pas de faire une longue litanie de projets individuels mais de montrer comment leur articulation permet de dégager un sens historique aux transformations ayant façonné la gestion des illégalismes depuis le XIXe siècle. Les instruments de régulation, destinés à produire de l'égalité de traitement en généralisant la mise en visibilité des illégalismes économiques et financiers, se sont succédés au rythme irrégulier des priorités politiques et des contextes de crise. Pourtant, il en ressort concrètement un processus de conversion-adaptation des modalités de différenciation entre illégalismes, (re)produisant de l'inégalité selon des enjeux de personne et de situation. Abordé dans la dernière partie du texte, l'avènement de la lutte anti-blanchiment comme nouveau socle de traitement de tous les capitaux associés à des activités illégales en est un des exemples les plus récents.

\section{Michel Foucault et le concept d'illégalismes}

\section{L'économie des illégalismes}

À la lecture de Surveiller et punir ainsi que des écrits ou interventions précédant et suivant de près cet ouvrage, l'expression d'économie des illégalismes est d'abord mobilisée pour rendre compte de la situation normative en France sous l'Ancien Régime. Foucault y dépeint une société aux prises avec une multitude d'illégalismes, dont une partie jouit d'espaces de tolérance. Chaque segment de la population y pratique un ensemble d'illégalismes et bénéficie de marges d'impunité variables. Parmi ces «marges d'illégalismes tolérés 13 », les représentants de la noblesse, du clergé et les grands propriétaires sont avant tout associés aux «illégalismes des privilégiés » dans la mesure où ils échappent « par statut, par tolérance, par exception, à la loi 14 ». Le contournement ou le détournement de règles douanières et commerciales constitue ensuite l'exemple type des «illégalismes bourgeois » souvent impuni. Les membres des couches populaires ont, quant à eux, dû conquérir leurs propres espaces de tolérance «par la force ou l'obstination » afin de survivre au quotidien ${ }^{15}$. Les mille et un «illégalismes populaires », tels que « la contrebande, le pâturage abusif, le ramassage de bois sur les terres du roi, quoique menacés de

12. Harcourt B., "Situation du cours », in M. Foucault, La société punitive, op. cit., Paris, Seuil/Gallimard, 2013, pp. 272-314.

13. Foucault M., Surveiller et punir, op. cit., p. 98.

14. Ibid., p. 146.

15. Ibid., p. 98. 
peines terribles, ne donnaient en réalité pratiquement jamais lieu à des poursuites 16 ». Certaines marges d'impunité sont octroyées volontairement par « consentement muet » quand d'autres reflètent davantage une négligence, un manque de vigilance ou «tout simplement l'impossibilité effective d'imposer la loi et de réprimer les infracteurs 17 ».

Plus important encore, le non-respect - toléré de fait - d'une série de lois et coutumes est décrit comme étant un élément fondamental de l'exercice du pouvoir sous l'Ancien Régime. Loin de toute univocité et de toute systématicité, l'économie des illégalismes est empreinte d'une application toute relative des normes royales et féodales. "Par exemple, la fraude fiscale la plus traditionnelle, mais également la contrebande la plus manifeste faisaient partie de la vie économique du royaume. Bref, il y avait entre la légalité et l'illégalité une perpétuelle transaction qui était l'une des conditions de fonctionnement du pouvoir à cette époque-là 18 ». Les illégalismes apparaissent moins sous le signe d'une pathologie sociale ou d'un dysfonctionnement perturbateur que comme un trait spécifique et indispensable de la vie économique et politique de la société. Ces pratiques sont inhérentes au maintien des équilibres et de l'ordre existant au sein du régime en étant ancrées au cour des activités routinières de la population ${ }^{19}$. La situation normative de l'Ancien Régime est marquée par cette coexistence complexe d'une multitude d'illégalismes plus ou moins installée et autorisée. Les membres des différentes strates sociales sont d'ailleurs prompts à défendre les largesses et négligences dont ils bénéficient. De manière ponctuelle, les actes de résistance plus ou moins visibles et plus ou moins violents à l'encontre de la levée de certaines taxes ont même pu fédérer des catégories très hétérogènes contre des droits féodaux communément vécus et dénoncés comme abusifs ${ }^{20}$. De la même façon, le contournement des règlements commerciaux passe régulièrement par un arrangement préalable entre marchands et artisans ${ }^{21}$. Une partie des refus fiscaux, des ententes commerciales et des pratiques de contrebande était aussi utile à la survie des plus pauvres qu'au développement économique de la bourgeoisie.

Dans cette configuration sociale particulière, ces deux grands segments de population que sont les couches populaires et la bourgeoisie se trouvent en situation d'interdépendance, parfois en tant qu'adversaires, souvent en tant qu'alliés de circonstance. D’un côté, les illégalismes populaires n'entrent pas nécessairement en confrontation directe avec les intérêts des commerçants issus de la bourgeoisie, puisqu'ils visent surtout à déjouer les droits féodaux.

16. Foucault M., «La prison vue par un philosophe français », in M. Foucault, Dits et écrits $I$. 1954-1975, Paris, Gallimard, 2001, p. 1596.

17. Foucault M., Surveiller et punir, op. cit., p. 98.

18. Foucault M., «Des supplices aux cellules », in M. Foucault, Dits et écrits I. 1954-1975, Paris, Gallimard, 2001, p. 1584.

19. Fischer N., Spire A., «L’État face aux illégalismes », Politix, 87-3, 2009, pp. 7-20.

20. Foucault M., Surveiller et punir, op. cit.

21. Gros F., «Foucault et la société punitive », Pouvoirs, 13, 2010, pp. 5-14. 
De l'autre, l'attitude de la bourgeoisie à l'égard de ces «illégalismes d'en bas » fluctue en fonction des intérêts en jeu. " [La bourgeoisie] soutient ces luttes anti-légales dans la mesure où elles la servent, elle les lâche quand celles-là tombent dans la criminalité de droit commun ou quand elles prennent la forme de luttes politiques. Elle accepte la contrebande, refuse le brigandage ; elle accepte le refus de l'impôt, mais refuse le pillage des diligences 22 ».

Michel Foucault dégage ici les traits d'une économie des illégalismes structurée autour d'une espèce de modus vivendi entre illégalismes bourgeois et illégalismes populaires. Cet état de relatif ajustement vole en éclat à mesure que l'Ancien Régime prend fin et que prend véritablement forme le capitalisme du XIXe siècle.

\section{La gestion différentielle des illégalismes}

À l'heure de l'essor du capitalisme industriel et du nouveau statut accordé à la propriété privée, les illégalismes populaires entrent en crise. Ils deviennent insupportables à la bourgeoisie lorsqu'elle accède à des positions économiques et politiques de premier plan. Foucault décrit par le menu cette recomposition de l'économie des illégalismes.

En zone rurale, « le resserrement des liens de propriété, ou plutôt le nouveau statut de la propriété terrienne et sa nouvelle exploitation, transforme en délits beaucoup d'illégalismes installés 23 ». La fin de l'Ancien Régime est marquée par l'abolition des droits féodaux, la disparition graduelle des terres communales et le morcellement de la propriété terrienne dont une partie significative est transferée vers la bourgeoisie. Avec la hausse des revenus fonciers, les nouveaux propriétaires multiplient les investissements tout en s'orientant vers une agriculture plus intensive. Débarrassés des charges féodales, ils bénéficient d'un droit de propriété désormais individualisé et absolu. Souhaitant maximiser l'usage de leurs terres et optimiser les revenus issus des produits agricoles, ils ne tolèrent plus les pratiques coutumières autrefois concédées à la paysannerie et ce, d'autant plus en période de pression démographique accrue. Ignorés par Foucault, les écrits de Karl Marx sur le «vol de bois » décrivent déjà la fin de ces concessions faites aux pauvres au moment où la relativité de la propriété féodale cède le pas à une conception absolutiste de la propriété privée ${ }^{24}$. Avec ce statut juridique de la propriété, le contournement des précédents édits et règlements est tranformé en atteintes aux biens. Ce type d'irrégularités étant parfois vital pour les couches les plus défavorisées de la société, sa remise en cause engendre « une série de réactions en chaîne, de plus en plus

22. Foucault M., La société punitive, op. cit., p. 147.

23. Foucault M., "La société punitive », in M. Foucault, Dits et écrits I. 1954-1975, Paris, Gallimard, 2001, p. 1335.

24. Voir l'édition critique de ces écrits : Lascoumes P., Zander H., Marx : $d u$ "vol de bois » à la critique du droit, Paris, Presses universitaires de France, 1984. 
illégales ou si on veut de plus en plus criminelles : bris de clôture, vol ou massacre de bétail, incendies, violences, assassinats 25 ». Il n'en demeure pas moins que les espaces de tolérance disparaissent pour les illégalismes populaires qui, avec la transformation de l'économie rurale, changent massivement de registre en passant « d'un illégalisme de fraude à un illégalisme de vol et de déprédation $26 »$.

Cette transformation est également liée au rapport entretenu avec la propriété industrielle et commerciale dans les espaces plus urbanisés. Désormais adossée au processus d'industrialisation et aux méthodes de production capitalistes, toute la fortune accumulée par la bourgeoisie (machines coûteuses, énormes stocks de matières premières et de produits finis) a été mise en contact permanent avec les forces productives (le prolétariat) ${ }^{27}$. Beaucoup de travailleurs sont passés du statut d'artisan à celui de salarié ou de journalier dans les grandes manufactures et dans les ports. De ce fait, ils n'ont généralement plus la propriété des outils et des matériaux avec lesquels ils travaillent et ont encore moins de prise sur les objets qu'ils produisent. Dépossédés des moyens de production, ils perdent toute opportunité et capacité de jouer avec les marges de la légalité via des illégalismes de fraudes dans la mesure où ils cessent d'être directement confrontés aux règles commerciales. Dans cette perspective, cibler le capital bourgeois dans sa matérialité devient pour eux la dernière forme d'illégalismes véritablement possible par le biais de rapines dans les usines et sur les docks. "Ainsi, par la force des choses, par l'installation du socle de l'économie capitaliste, ces couches populaires, se déplaçant de l'artisanat au salariat, sont en même temps obligées de se déplacer de la fraude au vol 28 ». Tandis que les profits dépendent de plus en plus du « corps à corps de l'ouvrier et de la marchandise 29 », la bourgeoisie craint l'augmentation du nombre de vols et d'attaques dans des proportions comparables à la taille inédite des nouveaux lieux de travail. Le développement de l'économie industrielle et l'évolution des modes de production sont doublés d'une intolérance systématique à l'égard des petits vols et autres pratiques transgressives telles que le ramassage des chutes de ferrail ou de fibres textiles.

Cette peur de la bourgeoisie n'est d'ailleurs pas uniquement focalisée sur les conditions de protection de la richesse matérielle entreposée dans les manufactures et les entrepôts. Le danger est aussi, et peut-être surtout, associé à la dissipation de la force de travail de l'ouvrier par l'ouvrier lui-même. En effet, l'attention portée aux conduites indisciplinées vise à circonscrire autant que possible les manifestations de l'immoralité ouvrière telles que l'alcoolisme, la paresse ou l'inexactitude, susceptibles d'entamer la productivité et

25. Foucault M., Surveiller et punir, op. cit., p. 101.

26. Gros F., «Foucault et la société punitive », op. cit., p. 10-11.

27. Ibid.

28. Foucault M., La société punitive, op. cit., p. 151.

29. Gros F., «Foucault et la société punitive », op. cit., p. 14. 
donc les profits escomptés ${ }^{30}$. Des préoccupations plus directement politiques sont également à l'œuvre afin d'éviter tout soulèvement populaire susceptible d'entrâner un nouvel élan révolutionnaire pouvant déstabiliser les équilibres économiques et sociopolitiques en place.

Sur le plan juridique, cette disparition des marges d'impunités dont bénéficiaient auparavant les couches populaires se concrétise par une pénalisation massive de leurs transgressions, rabattues du côté des «illégalismes de biens » à l'instar du « vol, comme illégalisme de celui qui produit à partir d'une matérialité qui ne lui appartient pas 31 ». Débarrassés des prédations féodales grâce à la Révolution française, les membres de la bourgeoisie se sont attelés à résorber les déprédations des couches populaires passées d'alliées de circonstance à adversaires désignés. «Et je crois que ce qui s'est passé, c'est que lorsque la bourgeoisie a pris le pouvoir politique et lorsqu'elle a pu adapter les structures d'exercice du pouvoir à ses intérêts économiques, l'illégalisme populaire qu'elle avait toléré et qui, en quelque sorte, avait trouvé dans l'Ancien Régime une espèce d'espace d'existence possible est devenu pour elle intolérable; et il a fallu absolument la museler. Et je crois que le système pénal, et surtout le système général de surveillance qui a été mis au point à la fin du XVIII siècle, au début du XIXe siècle, dans tous les pays d'Europe, c'est la sanction de ce fait nouveau : que le vieil illégalisme populaire qui était, dans certaines de ses formes, toléré sous l'Ancien Régime est devenu littéralement impossible : il a fallu effectivement mettre en surveillance généralisée toutes les couches populaires $32 »$. Plus qu'une simple crise des illégalismes populaires entérinée dans le droit pénal, Foucault donne surtout à voir la façon dont les contours de l'économie générale des illégalismes sont redessinés, aussi bien du côté des illégalismes que de leur gestion.

D’une part, la répartition sociale des illégalismes est désormais structurée autour d'une ligne de démarcation entre «illégalismes de biens » et «illégalismes de droits ». Comme nous l'avons noté, les groupes sociaux dominés se retrouvent déportés et cantonnés dans la première catégorie. À côté de ce type générique de conduites indisciplinées généralement associées aux non-possédants, les membres de la bourgeoisie se réservent un accès privilégié à l'autre catégorie, celle de la «fraude, comme illégalisme de celui pour qui la richesse est liée à la loi. Non pas qu'elle soit soumise à la loi mais elle donne accès à la possibilité de faire et défaire, d'imposer et de contourner la loi 33 ». Loin d'être réduits à la violation explicite de la loi, ils peuvent s'efforcer d'user du droit et de tourner la loi pour jouer activement avec les frontières démarquant le comportement conforme du comportement déviant. Dans cette optique et de manière plus récente, Doreen $\mathrm{McB}$ arnet a par exemple montré comment les

30. Foucault M., La société punitive, op. cit.

31. Ibid., p. 152.

32. Foucault M., «Des supplices aux cellules », op. cit., p. 1304.

33. Foucault M., La société punitive, op. cit., p. 152. 
élites économiques négociaient constamment avec ces frontières pour essayer d'éviter l'impôt et rester du côté de la légalité fiscale ${ }^{34}$.

D'autre part, la redistribution sociale des tâches transgressives est couplée à une réforme du système pénal, de l'appareil judiciaire et plus largement à une recomposition du droit positif au tournant du XVIII e et du XIX ${ }^{\mathrm{e}}$ siècle. Cette transformation des dispositifs juridiques contribue fortement à distinguer les modes de réaction sociale selon les types d’illégalismes concernés. Aux illégalismes de biens répondent l'attention continue de la police, les tribunaux ordinaires et la prison alors que les illégalismes de droits relèvent majoritairement d'une gestion discrète, complexe et dilatoire, dépendant de « juridictions spéciales avec transactions, accommodements, amendes atténuées, etc. 35 ». Les auteurs d'illégalismes de biens sont soumis à des dispositifs de contrôle et à des rituels de vérité se situant en dehors de leurs milieux d'appartenance et d'activités puisqu'ils relèvent du registre pénal. En revanche, les auteurs d'illégalismes de droits sont essentiellement jugés dans le cadre de rituels internes à leurs milieux professionnels tels que les tribunaux de commerce.

Cette recombinaison associée à une variété d'instruments et de voies de règlement juridiques entérine une triple différenciation révélatrice des rapports de pouvoir et de domination alors à l'œuvre. Elle recouvre une opposition de classe (illégalismes de biens/classes populaires vs illégalismes de droits/classe dirigeante) ainsi qu'une différence de qualification (catégorie pénale nette vs « catégorisation pluriforme et non-exclusivement pénale 36 ») et de traitement (procès/prison/publicisation vs juridictions spéciales/transactions/discrétion) ${ }^{37}$. Selon Jean-Claude Monod, c'est en cela que la « différenciation des illégalismes renvoie ainsi nettement à la domination de la classe qui a fait la loi » [...] «L'inégalité devant la justice ne tient pas seulement à des facteurs externes qu'évoquera également Foucault (le "prix" d'un avocat, la différence de traitement d'un individu par la police selon son statut, etc.), mais du système même des lois 38 ».

À cet égard, le choix de la réforme du droit et des dispositifs juridiques comme point d'entrée analytique vaut surtout comme indicateur des changements dans les modalités de pouvoir propres à un contexte socio-historique donné. Cette dimension juridique du pouvoir illustre un mode de domination sociale qui ne passe pas par une volonté de faire appliquer une loi uniforme mais par une gestion différenciée des comportements transgressifs ${ }^{39}$. Plutôt

34. McBarnet D., "Whiter than White Collar Crime: Tax, Fraud Insurance and the Management of Stigma”, The British Journal of Sociology, 42-3, 1991, pp. 323-344.

35. Foucault M., Surveiller et punir, op.cit., p. 104.

36. Lascoumes P., Les affaires ou l'art de l'ombre : les délinquances économiques et financières et leur contrôle, Paris, Centurion, 1986, p. 8.

37. Foucault M., Surveiller et punir, op. cit.

38. Monod J-C., Foucault, la police des conduites, Paris, Michalon, 1997, pp. 79-80.

39. Gros F., Michel Foucault, Paris, Presses universitaires de France, 2007. 
que de renvoyer à un appareil de répression et de suppression de tous les illégalismes, le système judiciaire contribue finalement à les organiser et à les maintenir dans un état d'équilibre économiquement utile et politiquement fécond 40 . Il vise à «établir entre eux une sorte de hiérarchie, de manière à en tolérer certains, à en punir d'autres, à en punir certains d'une manière, à punir les autres d'une autre manière 41 ». Fondamentalement et c'est là une de ses principales utilités, « la notion des illégalismes, c'est donc l'idée que la loi [est destinée] à gérer les marges de la légalité, qu'elle est un instrument de gestion 42 ». La domination ne se résume pas à la présence ou l'absence de contrôle et de sanction selon la position sociale des acteurs indisciplinés mais elle s'observe, de manière plus subtile, dans la distribution sociale des types d'illégalismes, dans l'existence d'espaces de jeux entre la norme écrite et la norme pratiquée et finalement dans la différence de réaction sociale. Ces éléments de réflexion sur ce moment de transformation historique et sur les formes de domination sociale ont depuis été repris pour alimenter des travaux empiriques questionnant l'économie des illégalismes et ce, par le biais d'objets de recherche marginaux en criminologie tels que la «criminalité d'affaires ».

\section{Le concept d'illégalismes pour étudier la criminalité d'affaires}

\section{La tension stratégique entre illégalismes et délinquance}

Pierre Lascoumes a inscrit une partie de ses travaux dans le prolongement du programme de recherche foucaldien consacré à la gestion différentielle des illégalismes. Il s'est intéressé aux réactions sociales suscitées par les pratiques de manipulation des règles du jeu économique et politique ${ }^{43}$. Pour ce faire, il a très tôt privilégié, avec d'autres chercheurs ancrés en criminologie, le syntagme de « criminalité d'affaires » permettant d'insister sur l'activité d'entreprises et sur les responsabilités collectives et ce, au détriment d'autres concepts de référence, que ce soit la notion de criminalité économique - jugée trop large - ou celle de criminalité en col blanc, minée par des débats définitionnels ayant en partie dilué sa valeur heuristique et sa force critique ${ }^{44}$. «La criminalité d'affaires telle que nous la concevons s'exerce au sein d'une activité commerciale, dans le but de détourner des circuits financiers et commerciaux ou d'échapper à des obligations légales. Elle porte sur des sommes importantes et repose le plus souvent sur une organisation impliquant d'autres agents (entreprises, banques, administration, organes politiques...) $)^{45}$ ».

40. Chantraine G., Salle G., «Pourquoi un dossier sur la "délinquance en col blanc" ? ", Champ pénal/Penal field [En ligne], vol. 10, 2013.

41. Brodeur J-P., " "Alternatives" à la prison : diffusion ou décroissance du contrôle social : une entrevue avec Michel Foucault », Criminologie, 26-1, 1993, p. 23.

42. Harcourt B., "Situation du cours », op. cit., p. 292.

43. Lascoumes P., Les affaires on l'art de l'ombre, op. cit.; Élites irrégulières : Essai sur la délinquance d'affaires, Paris, Gallimard, 1997 ; Corruptions, Paris, Presses de Sciences Po, 1999; Une démocratie corruptible, Paris, Seuil, 2011.

44. Kellens G., Lascoumes P., " Moralisme, juridisme et sacrilège : la criminalité d'affaires », 
Il a aussi et surtout délaissé l'opposition juridique entre légalité et illégalité. Calquer l'analyse sociologique et criminologique sur cette opposition juridique binaire aurait eu pour effet de passer sous silence les nombreux efforts consentis par les élites économiques et financières afin de déborder, déplacer voire remettre en cause les frontières mêmes de la légalité. "À côté de la légalité abstraite et verticale que l'on invoque toujours, s'immisce à tous niveaux une légalité horizontale, celle qui résulte des ajustements négociés par les acteurs pour faire coïncider leurs enjeux avec les règles $46 »$. Cet élément, pourtant au cœur de la vie des affaires, est souvent renvoyé dans l'ordre de l'impensé, sans véritable réflexion sur le jeu incessant avec les règles et les négociations entre les autorités du contrôle social et les entités visées à un moment donné. Or, la tension illégalismes-délinquance permet d'intégrer la part d'incertitude et de non-automaticité inhérente à l'application de la loi.

Alors que « le droit ne s'impose jamais de sa propre initiative aux faits sociaux 47 ", il convient de " concentrer la recherche non seulement sur la délinquance mais aussi sur le phénomène plus subtil d'évasion légale, non seulement sur le fiscal mais aussi de manière générale. Nous devons explorer la façon dont les élites économiques utilisent activement les moyens institutionnels, idéologiques et légaux pour jouir d'une immunité vis-à-vis du contrôle juridique 48 ». Ce parti pris épistémologique permet de couvrir un continuum allant des agissements condamnés aux usages « audacieux » et «créatifs » du droit en passant par les «tripotages astucieux » jusqu'aux comportements " théoriquement pénalisables mais pratiquement préservés par les difficultés de découverte de ces pratiques et par la faible part d'initiative des agences de contrôle 49 ». Cette démarche appelle à dépasser les apories des approches criminologiques tournées exclusivement vers l'étude des passages à l'acte délictueux, repérés et traités au niveau judiciaire, qui ne peuvent prétendre apporter une vue d'ensemble sur les illégalismes d'affaires. C'est aussi une façon d'évacuer l'idée que seuls les comportements incriminés par le droit pénal constituent des objets de recherche dignes d'intérêt criminologique ${ }^{50}$.

Déviance et société, 1-1, 1977, pp. 119-133. Pour un passage en revue des études et des débats académiques marquants sur la notion de criminalité en col blanc, voir Spire A., "Pour une approche sociologique de la délinquance en col blanc », op. cit.

45. Armand M. F., Lascoumes P., « Malaise et occultation: perceptions et pratiques du contrôle social de la délinquance d'affaires ", Déviance et société, 1-2, 1977, p. 149. De manière complémentaire et plus inclusive, Pierre Lascoumes et Carla Nagels ont récemment introduit l'expression de « déviance et délinquance des élites dirigeantes » pour désigner « des transactions de normes spécifiques liées à l'exercice d'une fonction de responsabilité privée ou publique et commises à titre individuel ou collectif par une instance dirigeante », in Lascoumes P., Nagels C., Sociologie des élites délinquantes. De la criminalité en col blanc à la corruption politique, Paris, Armand Colin, 2014, p. 15.

46. Lascoumes P., Élites irrégulières: Essai sur la délinquance d'affaires, op. cit., p. 16.

47. Ibid., p. 10.

48. McBarnet D., "Whiter than White Collar Crime: Tax, Fraud Insurance and the Management of Stigma”, op. cit., p. 342.

49. Lascoumes P., Élites irrégulières : Essai sur la délinquance d'affaires, op. cit., p. 239.

50. Tappan P., "Who is the criminal?", American Sociological Review, 12, 1947, pp. 96-102. 
En cela, la démarche de Lascoumes est proche de celle formalisée au cours des années 1940 par Edwin Sutherland dans ses écrits incontournables sur la criminalité en col blanc ${ }^{51}$. Celui-ci a notamment analysé la responsabilité des entreprises comme auteurs collectifs de trangressions et il a d'abord montré que des grandes firmes plusieurs fois pénalisées échappaient à l'image négative de la récidive en parvenant à neutraliser les effets du stigmate pénal. Ensuite, il est surtout allé au-delà des condamnations pénales dûment prononcées dans le monde des affaires pour s'intéresser aux processus orientant la qualification, le traitement et in fine le règlement des dossiers. Il a ainsi décrit une réaction sociale souvent éloignée de l'usage des instruments de régulation pénale au profit d'un recours massif aux procédures civiles, administratives ou professionnelles.

L'attention portée aux mécanismes de sélection des filières de traitement de ces dossiers lui a permis de saisir comment des actes pouvant relever de la justice pénale étaient finalement poursuivis devant d'autres juridictions et donc évacués des statisques sur la délinquance ${ }^{52}$. À partir de ce constat empiriquement fondé d'une mise à l'écart de la solution pénale, plusieurs chercheurs, dont Lascoumes, ont partagé l'ambition de Sutherland de ne pas s'en tenir à une simple posture de dénonciation. Peu fructueuse théoriquement, cette posture faisait surtout courir le risque de conclure, par syllogisme, à une " équité perverse » devant le pénal ${ }^{53}$, c'est-à-dire à une légitimation pour tous de pratiques répressives dont les effets disciplinaires étaient par ailleurs dénoncés. Inspirés par Foucault, nombreux étaient ceux à être davantage enclins à briser le bâton de la justice qu'à le tordre pour le retourner contre les fractions dominantes de la société comme ont pu le proposer des représentants de mouvements sociaux radicaux et des criminologues néo-marxistes ${ }^{54}$. Paradoxalement, la réflexion sur cette tendance à l'impunité pénale pour la criminalité d'affaires a mené au dépassement du pénalocentrisme en replaçant le pénal au sein d'un vaste ensemble de systèmes normatifs (civil, administratif, commercial, etc.) composant le droit positif. Comprendre comment un conflit particulier a été orienté vers une voie de règlement formelle autre que le droit pénal, quand bien même celui-ci était un code de lecture possible, passe par l'analyse des rapports complexes entre ces systèmes normatifs. Une telle analyse ouvre elle-même sur une problématique plus large que celle de l'impunité pénale, à savoir «l'organisation et le fonctionnement de la gestion différentielle des illégalismes dans une société 55 ».

51. Sutherland E., "Is white collar criminality a crime?", American Sociological Review, 10, 1945, pp. 132-139; White collar crime, Westport, Greenwood Press, 1949; White collar crime: the uncut version, New Haven, Yale University Press, 1983.

52. Voir sur ce point l'entretien réalisé avec Howard Becker dans ce numéro.

53. Acosta F., "À propos des illégalismes privilégiés. Réflexions conceptuelles et mise en contexte », Criminologie, 21-1, 1988, pp. 7-34.

54. Sur ce point, voir : Bérard J., "Tordre ou briser le bâton de la justice ? », Champ pénal/Penal field [En ligne], vol. 10, 2013; Nagels, C., «Les patrons en prison ? Une analyse des positions de criminologues néo-marxistes en matière de prise en charge de la délinquance des élites », in 
Insistant sur l'importance des travaux de Pierre Lascoumes dans l'émergence de cette nouvelle problématique à partir des débats sur l'impunité pénale, Fernando Acosta représente la gestion des illégalismes « sous la forme d'un réseau d'interactions entre divers systèmes normatifs relativement autonomes 56 ». À cette fin, il introduit une distinction entre «illégalismes typiques » et «illégalismes privilégiés». Alors que le mode de règlement des premiers appartient à un seul et unique système normatif (civil ou pénal ou administratif, etc.), la traduction juridique des seconds n'est pas d'emblée évidente dans la mesure où ils ont la particularité de pouvoir être appréhendés par plusieurs de ces systèmes normatifs. Dans cette perspective, les illégalismes populaires sont généralement confinés aux illégalismes typiques d'un système de régulation conduisant à une résolution "simpliste et expéditive 57 ». De leur côté, les illégalismes d'affaires correspondent fortement («mais non exclusivement») aux illégalismes privilégiés disposant d'un large éventail de modes de résolution, véritable condition de possibilité d'une gestion différenciée. "Parler de règlement différentiel, c'est au contraire mettre l'accent sur une pluralité des formes de règlement possibles et sur une pluralité des modes d'application. Pourquoi à tel moment tel conflit débouche-t-il sur telle forme de règlement et non sur telle autre est peut-être la question, apparemment simple, à laquelle nous essayons de répondre 58 ». L'enjeu porte ainsi sur la sélection du registre et de la qualification juridiques dont l'application effective dépend d'une série d'éléments dynamiques tels que la nature des rapports de force engagés entre auteurs suspectés d'illégalismes d'affaires et opérateurs du contrôle social. Ces rapports de force orientent aussi les solutions retenues pour régler un conflit, avec une place de choix réservée aux pratiques transactionnelles ${ }^{59}$, à l'image des amendes de plusieurs milliards de dollars payées aux États-Unis par des banques internationales pour éviter ou mettre fin à des poursuites judiciaires.

C'est précisément cet ensemble, marqué par une différenciation au sein même du droit et par des relations de pouvoirs portant sur le choix des modalités de traitement, que Lascoumes a nommé « système différentiel de régulation des illégalismes et infractions d'affaires $60 »$. La dimension juridique du pouvoir ne s'impose pas d'en « haut» mais se manifeste dans les interactions visant à qualifier des situations de conflit. «En matière juridique, être en situa-

A. Jaspart, S. Smeets, V, Strimelle et F. Vanhamme (eds.), "Justice! » Des mondes et des visions, Éditions online Erudit, Coll. Livres et Actes, 2014, pp. 106-120.

55. Acosta F., "À propos des illégalismes privilégiés. Réflexions conceptuelles et mise en contexte ", op. cit., p. 11.

56. Ibid., p. 12.

57. Ibid.

58. Lascoumes P., Les affaires on l'art de l'ombre, op. cit., p. 64.

59. Serverin E., Lascoumes, P. et T. Lambert, Transactions et pratiques transactionnelles, Paris, Economica, 1987.

60. Lascoumes P., Les affaires on l'art de l'ombre, op. cit., p. 84. 
tion de pouvoir, c'est avoir et garder la maîtrise des règles applicables dans les processus où l'on se trouve impliqué. Inversement, être en situation de dominé, c'est de se voir imposer une identité d'action, demeurer sans prise sur le déroulement des interactions 61 ». Cette possibilité pour les illégalismes d'affaires d'être arrimés à différents systèmes normatifs a souvent pour effet concret de faire obstacle à l'application des modalités les plus contraignantes, à savoir le choix des poursuites pénales, que ce soit au début du dossier ou au fil de son cheminement ${ }^{62}$.

À cet égard, il n'y a semble-t-il qu'un pas à franchir pour établir un lien, serait-il implicite, entre les conclusions énoncées par Sutherland, puis celles proposées par Foucault une vingtaine d'années plus tard. Lascoumes donne d'ailleurs une lecture pour le moins foucaldienne de l'ouvrage clé du sociologue/criminologue américain portant «sur le traitement différentiel de la délinquance d'affaires par rapport aux délinquances dites “ordinaires”. Le "WCC" [White Collar Crime] bénéficie, montre-t-il [Sutherland], de modes de règlement privilégiés par rapport à la délinquance des classes populaires 63 ». Sutherland s'évertuait à montrer que ces "privilèges » accordés à la criminalité en col blanc, sous la forme d'une réaction sociale généralement plus faible et plus discrète que pour la criminalité « ordinaire », n'était aucunement corrélé au degré de gravité des atteintes et dommages causés. Il dénonçait plutôt ce que d'aucuns ont qualifié de « cercle vicieux qui s'installe entre faible sanction et faible perception de gravité, permettant d'échapper au stéréotype du criminel 64 ». Articulant cette double filiation intellectuelle Sutherland-Foucault, c'est au travers de la tension entre illégalismes et délinquance que Lascoumes travaille et reformule la question de l'écart existant entre la multiplicité des comportements transgressifs caractérisant la vie des affaires et la rareté des condamnations judiciaires en la matière. " À certains moments de leur carrière (pour traverser une situation de crise financière ou un dur conflit du travail), ou bien dans le cadre de certains pans de leurs activités (conclusions de marchés étrangers, maîtrise de concurrents menaçants), des entreprises, y compris parmi les plus prospères et les plus cotées, peuvent développer des activités transgressant des normes légales. Mais ce n’est pas pour autant que l'on peut automatiquement parler de "délinquance d'affaires”. En effet, le droit est un système de codage mobilisable pour nommer

61. Lascoumes P., «Foucault et les sciences humaines, un rapport de biais : l'exemple de la sociologie du droit », Criminologie, 26-1, 1993, p. 50.

62. Lascoumes P., Moreau-Capdevielle G., «Des finesses de citadins à la délinquance des sociétés commerciales : place de la justice pénale dans le contrôle de la délinquance des affaires », Revue de science criminelle et de droit pénal comparé, 4, 1984, pp. 707-722 ; Acosta F., « De l'événement à l'infraction : le processus de mise en forme pénale », Déviance et société, 11-1, 1987, pp. 1-40.

63. Lascoumes $P$., « Le traitement social de la délinquance économique et financière : cataplasme ou antibiotique? ", Les cabiers de la sécurité, 36, 1999, p. 188.

64. Kellens G., Lascoumes P., « Moralisme, juridisme et sacrilège: la criminalité d'affaires », op. cit., p. 130; Chapman D., Sociology and the stereotype of the criminal, Londres, Tavistock, 1968. 
les situations de conflits de valeurs et d'intérêts et pour les mettre en forme en vue d'un règlement. On parlera de figure de délinquance lorsqu'une qualification pénale parvient à être appliquée avec succès à la situation. On parlera d'illégalisme quand la pratique concernée résiste ou échappe à l'application du code juridique, soit parce que cette pratique est restée sans visibilité sociale, soit parce qu'aucun acteur social (victime ou agence de contrôle) n'a su ou pu appliquer ce code en raison de la résistance de l'auteur 65 ».

Malgré bon nombre d'intuitions et de réflexions convergentes, un point de désaccord distingue toute de même la réflexion initiée par Sutherland de celle développée par Lascoumes pour comprendre les comportements transgressifs et leur régulation dans le monde des affaires. "Le radicalisme de Sutherland présente cependant certaines limites. Les infractions liées à la vie des affaires sont pour lui un dysfonctionnement social, un signe de désorganisation qui se répandrait faute de régulation adaptée $66 »$. Un optimisme latent se dégagerait ainsi de l'analyse de Sutherland avec une croyance en la capacité régulatrice du droit sans bouleverser outre mesure l'ordre économique et financier en place. Or, les illégalismes d'affaires étant une composante à part entière de cet ordre particulier, multiplier de façon plus ou moins spectaculaire le nombre de règlements, augmenter les moyens de contrôle et durcir les sanctions sans pour autant modifier la situation générale est accueillie avec un certain scepticisme par Lascoumes. Cette vision est particulièrement frappante dans l'ouvrage sur le Capitalisme clandestin, co-écrit avec Thierry Godefroy, au sous-titre évocateur : l'illusoire régulation des places offshore 67 . À rebours du sens commun réduisant ces places à l'état de parasites périphériques au système financier, ils montrent comment les pratiques associées à ces « moutons noirs » de l'économie capitaliste sont désormais au cœur même du fonctionnement contemporain du commerce et de la finance internationales ${ }^{68}$. À ce titre, leur rôle essentiel dans la circulation actuelle des flux de capitaux transnationaux rend la réalité de leur régulation illusoire sans questionner le système de pratiques financières d'une manière structurelle.

In fine, Lascoumes défend et précise l'hypothèse de tranformation historique proposée par Michel Foucault. «Il n'y a pas eu, comme on le croit souvent, hyper-contrôle d'un côté, occultation totale de l'autre. La situation est plus complexe. S'il y a bien eu tentative générale de mise en visibilité de tous les illégalismes au XIXe siècle, celle-ci s'est faite de façon différentielle et selon des modes hétérogènes. Une différence majeure sépare la mise en visibilité des uns et des autres. Son siège est à rechercher dans la forme des instruments de

65. Lascoumes P., Élites irrégulières : Essai sur la délinquance d'affaires, op. cit., p. 232-233.

66. Lascoumes $\mathrm{P}$., "Le traitement social de la délinquance économique et financière : cataplasme ou antibiotique? ", op. cit., p. 189-190.

67. Godefroy T., Lascoumes P., Le capitalisme clandestin : l'illusoire régulation des places offshore, Paris, Éditions la Découverte, 2004.

68. Voir aussi : Chavagneux C., Murphy R. et R. Palan, Tax Havens: How Globalization Really Works, London, Cornell University Press, 2010. 
régulation utilisés 69 ». Rapportée à la criminalité d'affaires, « l'euphémisation des conflits marquant la vie des affaires s'opère donc par un jeu différentiel de règles et de procédures de règlement qui préservent, pour l'essentiel, les intérêts de la bourgeoisie et de la petite bourgeoisie d'affaires. Si ces dernières ont toujours à craindre les réverbères, elles peuvent le plus souvent être satisfaites des lucioles et lumignons qui régulent la plupart de leurs différends. L'essentiel demeure dans l'ombre 70 ». Ces citations datent de près de trente ans et leur auteur n'a pas manqué d'insister sur l'évolution des modalités de perception de cette forme de criminalité au sein de l'opinion publique et des institutions judiciaires avec un déplacement sensible de la lumière des réverbères ${ }^{71}$. Les transformations ayant marqué le corps de la magistrature, ses relations avec d'autres acteurs sociaux (journalistes, etc.) et l'action des juges ont aussi contribué à la prise en charge judiciaire des illégalismes d'affaires au sortir des années 1980 et au cours des années 1990 72. Soutenu par des scandales à répétition, ce pic d'attention a toutefois été suivi d'une période de réduction continue des moyens octroyés aux dispositifs de contrôle spécifiques ${ }^{73}$; à l'exception notable des juridictions interrégionales spécialisées (JIRS) créées en 2004 dont le domaine d'intervention couvre certes les « infractions économiques et financières » mais aussi la «criminalité organisée ». Cette tendance générale a été couplée au mouvement de fond vers la dépénalisation du droit des affaires ${ }^{74}$. Si ce moment d'atonie est en partie brisé à la faveur du retour cyclique des scandales dans un contexte de crise, il convient d'étudier l'opérationnalisation des réformes adoptées - comme la création en 2013-2014 d'un nouveau parquet national financier dédié à la lutte contre la délinquance financière et la fraude fiscale ainsi que d'un nouvel Office central de lutte contre la corruption et les infractions financières et fiscales - avant de questionner l'actualité des citations ci-dessus.

Un fait peut tout de même être confirmé. Les illégalismes de droits ont cessé d'être monopolisés par les groupes sociaux dominants, en raison des transformations relatives à l'interventionnisme étatique dans les pays occidentaux depuis la fin des années 198075.

69. Lascoumes P., Les affaires on l'art de l'ombre, op. cit, p. 8-9.

70. Ibid., p. 255.

71. Lascoumes P., Élites irrégulières: Essai sur la délinquance d'affaires, op. cit.

72. Roussel V., Affaires de juges. Les magistrats dans les scandales politiques en France, Paris, Éditions La Découverte, 2002.

73. Godefroy T., «La délinquance économique et financière serait-elle en voie de disparition? », Après-demain, 16, 2010, pp. 31-34.

74. Ibid. ; Snider L., "The Sociology of Corporate Crime: An Obituary", Theoretical Criminology, 4-2, 2000, pp. 169-206.

75. Fischer N., Spire, A., "L’État face aux illégalismes », op. cit. 


\section{Une problématique foucaldienne revisitée}

\section{La sphère judiciaire au sein de l'univers bureaucratique}

Depuis plusieurs années maintenant, quelques sociologues et politistes ont revisité le programme de travail associé au concept d'illégalismes à lumière de problématiques de recherche renouvelées. "Notre intention ici est de revenir sur la notion de "gestion différentielle des illégalismes" en la considérant non pas comme le privilège d'une classe sur les autres mais comme un mode de domination assuré par des agents de l'État, en particulier lorsque ceux-ci sont confrontés à des pratiques illicites qui ne visent pas les biens ou les personnes mais la transgression de lois ou de règlements. Au cours des années 1980, l'émergence d'un discours international sur les Droits de l'Homme a conduit à une reconfiguration des modalités de contrôle des États démocratiques sur les populations. Dans le contexte du tournant de la rigueur opéré aux États-Unis puis dans la plupart des pays européens, le thème de l'État de droit est opportunément venu se substituer aux objectifs d'un développement de l'État social en perte de légitimité. Des acteurs non gouvernementaux, au premier rang desquels on trouve les immigrants et les réfugiés, ont ainsi pu devenir des sujets de droit international. Parallèlement, l'intensification de la mobilité des biens comme des personnes a fortement contribué à déborder la capacité régulatrice des États-nations qui ont alors dû redéployer leurs dispositifs d'intervention, en se concentrant davantage sur la pénalisation de l'immigration irrégulière et plus généralement sur le contrôle de l'ensemble des populations pauvres. Dans la période contemporaine, les “illégalismes de droit” ne sont donc plus l'apanage des dominants : ils concernent plus généralement toutes les catégories d'individus aux prises avec l'État (étrangers en situation irrégulière, détenus, contribuables ou bénéficiaires de prestations sociales). La question de la régulation de ces illégalismes ne se conçoit plus seulement par opposition aux “illégalismes de biens”, mais se pose à l'intérieur d'un espace où toutes les catégories sociales de la société sont susceptibles d'être représentées 76 ».

Ces auteurs ont contribué à amplifier un changement de perspective analytique dans l'étude empirique de la gestion des illégalismes. Au travers de l'objet de recherche "criminalité d'affaires », Pierre Lascoumes avait surtout cherché, du moins initialement, à mettre à jour « des systèmes de pouvoir idéologique et répressif sur lesquels repose la justice criminelle 77 ». Sans nier l'importance de l'institution judiciaire et de ses acteurs, Fischer et Spire ont invité à déplacer le regard sociologique « de la sphère judiciaire vers l'univers bureaucratique » en resituant l'analyse sur le travail quotidien d'agents de

76. Ibid., p. 11.

77. Armand M. F., Lascoumes P., « Malaise et occultation: perceptions et pratiques du contrôle social de la délinquance d'affaires », op. cit., p. 166. 
l’État (autres que les magistrats) en prise directe avec des auteurs d'illégalismes. À partir de leurs travaux sur les conditions sociales d'application du droit par les fonctionnaires de terrain, la gestion des illégalismes est toujours appréhendée comme une activité relationnelle, au travers des rapports de force entre ces acteurs du contrôle social et leur " public » spécifique. Ces relations de pouvoir sont étudiées dans une grande variété de situations, qu'il s'agisse des agents de l'administration fiscale aux prises avec les stratégies d'évitement fiscal des contribuables, des gardes-frontières traitant des populations en mouvement, des douaniers gérant des flux de marchandises tout en traquant les tentatives de fraude, de contrebande et de contrefaçon ou encore des fonctionnaires municipaux faisant face aux usages du squat comme autant d'arrangements entre les règles de propriété et les droits au logement ${ }^{78}$.

Si ces travaux montrent bien que les illégalismes de droits ne sont plus restreints à un groupe social spécifique, leurs auteurs n'en concluent pas pour autant à la disparition d'une gestion différentielle selon les enjeux de situation et de personne, loin s'en faut. Les écrits d'Alexis Spire sur l'application quotidienne du droit fiscal en France en sont une démonstration éclairante.

\section{La gestion différentielle entre illégalismes fiscaux}

Avec $15 \%$ de ménages imposés sur le revenu jusqu’aux années 1950, les contrôles fiscaux ont d'abord pesé sur les résidents les plus aisés dans la période contemporaine. Depuis lors, la situation a radicalement changé ${ }^{79}$. D’un côté, la part des ménages soumis à l'impôt direct a fluctué au gré des orientations politiques pour aujourd'hui demeurer autour des $50 \%$. De l'autre, les réformes ayant affermi le développement de l'État providence ont paradoxalement mis la bureaucratie fiscale en contact avec l'ensemble de la population, y compris les usagers non-imposés, via la redistribution des prestations sociales. Celles-ci étant allouées sous condition de ressources, elles sont attribuées en fonction du certificat d'imposition ou de non-imposition delivré par cette administration. Au cours des quinze dernières années, les agents des finances publiques ont donc continué à collecter les taxes d'État

78. Aguilera T., «Gouverner les illégalismes. Les politiques urbaines face aux squats à Paris », Gouvernement et action publique, 3-3, 2012, pp. 101-124 ; Chantraine G., Salle G., " Le droit emprisonné ? Sociologie des usages sociaux du droit en prison », Politix, 87-3, 2009, pp. 93107 ; Chauvin S., «En attendant les papiers. L'affiliation bridée des migrants irréguliers aux États-Unis ? ", Politix, 87-3, pp. 47-69; Favarel-Garrigues G., La police des moeurs économiques : de l'URSS à la Russie (1965-1995), Paris, CNRS Éditions, 2007 ; Fischer N., "Une frontière "négociée". L'assistance juridique associative aux étrangers placés en rétention administrative ", Politix, 87-3, 2009, pp. 71-92 ; Heyman J., « Risque et confiance dans le contrôle des frontières américaines ", Politix, 87-3, pp. 21-46 ; Moreau de Bellaing C., «Violences illégitimes et publicité de l'action policière », Politix, 87-3, 2009, pp. 119-141; Spire A., Faibles et puissants face à l'impôt, Paris, Éditions Raisons d'agir, 2012 ; « La domestication de l'impôt par les classes dominantes ", Actes de la recherche en sciences sociales, 190, 2011, pp. 58-71 ; "Échapper à l'impôt ? La gestion différentielle des illégalismes fiscaux ", Politix, 87-3, 2009, pp. 143-165.

79. Spire A., Faibles et puissants face à l'impôt, op. cit. 
tout en participant à cette redistribution des prestations sociales, notamment en direction des ménages non-imposables 80 . Ainsi, l'administration fiscale s'est retrouvée à occuper une position centrale au sein du dispositif de contrôle des bénéficiaires de telles prestations.

Toutes les catégories sociales sont dès lors confrontées, à des degrés divers, aux règles de la bureaucratie fiscale. À ce titre, une écrasante majorité, si ce n'est la totalité des ménages, se trouve de facto en situation de pratiquer des formes d'illégalismes de droits touchant à la fiscalité. En principe, toutes les transgressions fiscales sont contrôlées, traitées et sanctionnées de manière égalitaire. En pratique, Spire souligne l'absence d'uniformité dans les conditions sociales d'application du droit fiscal et dévoile par là-même un mode de (re)production d'inégalités devant l'impôt. Pour rendre compte de cette gestion différentielle des illégalismes fiscaux au sein des centres d'impôts, il a mené des entretiens auprès d'agents du fisc et observé leurs interactions avec des usagers venant plaider leur cause. Loin d'un prétendu complot ourdi au plus haut niveau de l'État et partagé secrètement par ses agents, Spire ambitionne plutôt de résoudre l'énigme suivante : "comment une institution composée d'agents soucieux d'œuvrer pour l'intérêt général et le bien commun peut-elle (re)produire autant d'inégalités ? 81 ». En effet, les face-à-face orchestrés entre ces agents et « leur » public laissent entrevoir des réactions différenciées selon les catégories d'impôts et de contribuables concernées avec des inégalités pour partie liées aux modalités de contrôle et aux injonctions hiérarchiques en vigueur.

Premièrement, l'informatisation du contrôle fiscal dans les années 1990 a contribué à accentuer les différences de traitement entre illégalismes fiscaux. Ce processus a accru les capacités de vérification des déclarations de ressources - relatives à certains types de prélèvements et de revenus - pouvant être recoupées avec des données d'ores et déjà communiquées par des employeurs ou des caisses de paiement (celles des pensions de retraites ou des minima sociaux, par exemple). Dans ces cas, les agents de l'administration fiscale, assis derrière leur ordinateur, sont en mesure de comparer les revenus déclarés avec les montants officiellement versés par les organismes-payeurs ${ }^{82}$. En revanche, ces ressources informatiques sont bien moins efficaces pour contrôler les revenus des non-salariés tels que les entreprises, les professions libérales et les travailleurs indépendants disposant d'une capacité accrue de dissimulation. Repérer les transgressions fiscales parmi les contribuables les plus aisés est également une tâche ardue puisque les déclarations des ménages assujettis à l'impôt de solidarité sur la fortune ne peuvent être recoupées avec d'autres données. Dans cette situation d'auto-déclaration, l'agent de contrôle est largement tributaire des informations transmises par le contribuable. Alors

80. Ibid.

81. Ibid., p. 9.

82. Ibid. 
que l'absence de correspondance entre revenu déclaré et revenu imposable est relativement aisée à détecter dans certains cas et pour certains contribuables, les transgressions propres à d'autres prélèvements offrent bien plus de résistances au repérage social, et davantage d'opportunités pour jouer avec les marges du droit et abuser du système fiscal.

L'émergence du débat politique sur la « fraude sociale » à partir du milieu des années 1990 a eu pour effet d'étendre cette surveillance informatisée aux groupes sociaux non-imposables. La multiplication des échanges d'informations entre les services fiscaux et l'assurance-maladie, Pôle emploi ou encore les caisses d'allocations familiales est venue renforcer les efforts déployés à l'encontre des fraudes aux prestations sociales. Par exemple, le croisement des bases de données permet de comparer les informations dont dispose l'administration fiscale avec les données reçues au sein des antennes de Pôle emploi. Si, dans sa déclaration trimestrielle obligatoire, un allocataire du revenu de solidarité active (RSA) certifie avoir été sans ressources lors des trois derniers mois alors qu'il a bien été remunéré sous forme de salaires durant la période écoulée, l'agent du Pôle emploi sera armé pour repérer la fraude. En revanche, les ménages redevables de l'ISF ne font pas l'objet d'un tel suivi puisque les demandes d'interconnexion d'autres fichiers potentiellement pertinents ( $\mathrm{du}$ cadastre, des assujettis à la taxe d'habitation, etc.) sont restées lettre morte ${ }^{83}$.

Deuxièmement, les directives internes adressées aux agents de l'administration fiscale les ont fortement orientés vers des logiques de négociation et de régularisation plutôt que vers des procédures contentieuses dont la durée et les chances de succès demeurent aléatoires ${ }^{84}$. Ici, l'expression de " gestion différentielle » est particulièrement pertinente dans la mesure où les agents de l'État sont incités à varier leur répertoire d'actions avec une priorité donnée aux instances de conciliation au nom d'un pragmatisme prenant le pas sur le légalisme ${ }^{85}$. La place accordée à ces espaces de négociation et au discernement des agents bénéficie très souvent aux contribuables disposant des ressources suffisantes pour faire appel aux services de conseillers spécialisés afin de gérer au mieux l'interaction avec un représentant de l'administration fiscale. Les membres des classes moyennes et populaires ne disposent pas des capitaux permettant d'accéder à ce savoir-expert pour se familiariser avec les subtilités $\mathrm{du}$ droit fiscal et ses 4000 articles. Ils dépendent davantage des cellules d'informations du fisc que des avocats fiscalistes pour obtenir de l'aide dans ce domaine. Et si les fonctionnaires peuvent bien évidemment répondre aux demandes des ménages modestes, ils restent quand même, en dernière instance, juges et parties dans l'évaluation des requêtes formulées par ces contribuables. Spire montre comment les inégalités de ressources placent les usagers contribuables dans des positions différentes pour rester du côté de la légalité

83. Ibid.

84. Spire A., «Échapper à l’impôt ? La gestion différentielle des illégalismes fiscaux », op. cit.

85. Ibid. 
et trouver l'équilibre entre « dissimulation tolérée » et « maquillage frauduleux $» 86$.

Les variations dans les modes de contrôle, la technicité de la matière fiscale, la place accordée aux procédures de négociation, les évolutions législatives, les injonctions hiérarchiques et la façon dont elles sont mobilisées par les «street level bureaucrats » sont autant d'éléments traduisant et reproduisant les inégalités statutaires entre les citoyens. «L'approche sociologique des inégalités devant l'impôt incite donc à distinguer les illégalismes populaires qui consistent à transgresser explicitement la loi en s'exposant au contrôle de l'administration et les illégalismes plus répandus chez les dominants, qui consistent à utiliser les failles du droit en affichant “sa bonne foi” 87 ». Ainsi décrite, la gestion des illégalimes fiscaux apparât sous les traits d' " un régime complexe de dispositifs dérogatoires et de traitement différentiels » qui remet en cause le principe d'égalité de traitement entre usagers devant une administration publique ${ }^{88}$. "Tout comme dans le cas de l'école, la force de ce mécanisme [de (re)production d'inégalités sociales] est de parvenir à se développer en grande partie à l'insu des agents qui le mettent en œuvre 89 ».

Une autre dynamique est à l'œuvre dans les usages des instruments de régulation « anti-blanchiment » avec la gestion d'une multitude d'illégalismes de biens et de droits, y compris fiscaux, couverts par le dispositif de lutte contre l'argent sale. En effet, l'inclusion récente de la fraude fiscale au sein de ce dispositif signifie qu'un même instrument de régulation est officiellement mobilisable pour cibler toutes les formes de flux financiers illicites, quels que soient les enjeux de personne et de situation. Malgré cette indifférenciation inscrite dans la loi, les conditions sociales d'interprétation et d'appropriation de cet instrument tendent à maintenir une gestion différentielle des illégalismes fiscaux.

\section{Illégalismes de biens, illégalismes de droits et argent sale}

\section{Un instrument de régulation contre toutes les formes de délinquance finan- cière?}

Hier limitées aux produits des trafics de stupéfiants, les normes de référence contre le blanchiment d'argent et les prérogatives allouées aux cellules nationales de renseignement financier sont aujourd'hui présentées comme le cadre privilégié pour surveiller et punir toutes les formes de « délinquance financière 90 ». En France, la prise en compte de la fraude fiscale au sein de ce

86. Spire A., Faibles et puissants face à l'impôt, op. cit.

87. Ibid., p. 12.

88. Spire A., «Échapper à l'impôt ? La gestion différentielle des illégalismes fiscaux », op. cit.

89. Spire A., Faibles et puissants face à l’impôt, op. cit., p. 13.

90. Mobilisée sous l'égide la Commission européenne, la notion criminologique de délinquance financière y recouvre les enjeux de blanchiment de capitaux et de financement du terrorisme : 
dispositif illustre parfaitement cette narration officielle portée par le directeur de la cellule de renseignement financier, Tracfin. «Le dispositif, qui a conduit, sous l'égide du Gafi [Groupe d'action financière], à la création des cellules de renseignement financier, a toujours su montrer son efficacité et sa flexibilité : initialement conçu pour la seule lutte contre le financement du trafic de drogue, il a progressivement été élargi au combat contre toutes les formes de flux financiers illicites, ainsi que contre le financement du terrorisme. La crise économique et financière qui sévit depuis plusieurs années amène à une réflexion nouvelle sur le nécessaire renforcement des instruments de régulation dans le domaine financier et il est probable que cette réflexion conduira à conférer un rôle renforcé à ces structures originales dont la fonction de surveillance des flux financiers s'est imposée comme un corollaire indispensable à leur libéralisation ${ }^{91}$ ».

Blanchiment de tous crimes ou délits ; travail dissimulé ; travail illégal ; abus de confiance; abus de biens sociaux; escroquerie; escroquerie en bande organisée ; exercice illégal de la profession de banquier; abus de faiblesse ; infractions fiscales (fraude, déclaration absente ou fausse, organisation frauduleuse d'insolvabilité); recel ; détournement de fonds ; faux et usage de faux ; proxénétisme; banqueroute; vol ; infraction à la législation des stupéfiants ; corruption ; infractions douanières ; contrefaçon ; corruption d'agent public étranger ; trafic d'influence; vol en bande organisée ; financement du terrorisme ; association de malfaiteur ; exploitation illicite de cercle de jeux ; concussion; perception ou exonération indue de sommes par une personne exerçant une fonction publique. Sous ses airs d'inventaire à la Prévert, cette liste recense l'ensemble des infractions sur lesquelles ont récemment enquêté les agents de Tracfin en raison de soupçons de blanchiment de capitaux ou de financement du terrorisme ${ }^{92}$. Elle balaie un large spectre de conduites, avec des illégalismes de biens tels que le vol et des illégalismes de droits tels que la fraude fiscale. En sus du financement d'actes terroristes et des agissements visant à déguiser l'origine de capitaux issus d'activités criminelles, la lutte contre l'argent sale tend donc à recouvrir la dissimulation de l'existence ou de la nature de revenus généralement obtenus légalement mais illégalement soustraits aux règles d'imposition. "On considère qu'une infraction de blanchiment de capitaux (article 1er, point 2) est commise lorsque le blanchiment porte sur les produits d'une "activité criminelle" sous-jacente. [...] Les nouvelles normes du Gafi considèrent les "infractions fiscales pénales" (liées aux impôts directs et indirects) comme des infractions sous-jacentes ${ }^{93}$ ». Avec cette entrée dans le périmètre de l'anti-blanchiment, la fraude fiscale est deve-

http://ec.europa.eu/internal_market/company/financial-crime/index_en.htm

91. Tracfin, Rapport annuel 2011, Paris, 2012, p. 3.

92. Ibid.

93. Commission européenne, Rapport de la Commission européenne au Parlement européen et au Conseil concernant concernant l'application de la directive 2005/60/CE du Parlement européen et $d u$ Conseil relative à la prévention de l'utilisation $d u$ système financier aux fins $d u$ blanchiment de capitaux et du financement du terrorisme, Bruxelles, 2012, p. 4. 
nue synonyme d'argent sale. En considérant que l'objectif du blanchiment, en tant qu'opération incriminée, est précisément de faire ressortir de l'argent « propre » du circuit, c'est effectivement partir du principe qu'au départ, l'argent est bel et bien entaché, qu'il est sale.

«L’Union européenne est à la pointe des efforts déployés au niveau international pour combattre le blanchiment des produits du crime. Les flux d'argent sale peuvent fragiliser la stabilité du secteur financier et ternir sa réputation, tandis que le terrorisme ébranle les fondements mêmes de notre société 94 ». Mobilisée dans les textes européens et internationaux en la matière, l'expression stigmatisante d'argent sale et ce qu'elle recouvre officiellement n'ont rien d'évident, d'objectif ni de figé. Le contenant comme le contenu renvoient à un processus continu de construction et de redéfinition de certaines frontières sociales par les parties intéressées ${ }^{95}$. Du «terrorisme » à la « fraude fiscale », le dégradé d'illégalismes couvert par la notion d'argent sale et donc par le dispositif de surveillance et de renseignement financier est aujourd'hui d'une ampleur sans précédent. Si le directeur de Tracfin peut désormais arguer que sa cellule est au cœur d'un « combat contre toutes les formes de flux financiers illicites ", c'est au regard de ce champ d'application inédit. Discutée au sein de l'Union européenne avec le projet de 4 e directive anti-blanchiment élaboré en 2012-13 mais déjà actée en France depuis fin 2009, la prise en compte de la fraude fiscale s'ajoute à toute une gamme de pratiques illégales d'acquisition et/ou de rétention de capitaux. Aussi bien l'origine que la destination des fonds peuvent servir à qualifier leur « saleté » et permettre d'engager des poursuites pénales à la suite de leur repérage.

Cette inclusion des questions fiscales entre plus largement en résonnance avec la crise financière internationale ayant engendré dès 2008, 2009 de nombreuses discussions, dans les fora internationaux type G7/8, Gafi et G20, autour de la stabilité du système financier. Au regard de la situation économique, les appels à renforcer la régulation se sont multipliés, notamment à l'approche des réunions du G20 qui s'est alors imposé, médiatiquement si ce n'est diplomatiquement, comme «l'instance principale de concertation économique internationale 96 ». Dans ce cadre, le «Conseil européen de prin-

94. Commission européenne, Communiqué de presse. Lutte contre le blanchiment de capitaux: des règles renforcées pour contrer les nouvelles menaces, Bruxelles, 2013, p. 1.

95. Peebles G., "Filth and lucre: the dirty money complex as a taxation regime”, Anthropological Quarterly, 85-4, 2012, pp. 1229-1256.

96. Etabli en 1999 après le sommet de Cologne du G7 et en réponse à la crise asiatique de 1997, le G20 vise officiellement à regrouper les « grandes économies avancées » et les «économies émergentes » afin d'évoquer les questions relatives à la stabilité financière internationale. Il se compose des pays membres du G7, de l’Union européenne (via la présidence du Conseil et la Banque centrale européenne), de l'Afrique du Sud, de l'Arabie Saoudite, de l'Argentine, de l'Australie, du Brésil, de la Chine, de la Corée du Sud, de l'Inde, de l'Indonésie, du Mexique, de la Russie et de la Turquie. Cf. Sgard, J., « Le G20 et la régulation financière », in PostelVinay K. (ed.), Le G20, quoi, qui, comment ?, Dossier du CERI, 2010. Disponible sur : http://www.ceri-sciencespo.com/cerifr/kiosque.php 
temps » 2009 (19-20 mars) avait justement été l'occasion, pour les chefs d'État et de gouvernement de l'UE, d'adopter une position commune en vue du sommet du G20 prévu à Londres dix jours plus tard. Dans ce contexte de crise, ils avaient invité à « combattre avec détermination la fraude fiscale, la criminalité financière, le blanchiment d'argent et le financement du terrorisme [...] ainsi que toute menace à la stabilité financière et à l'intégrité du marché 97 ».

D’un dispositif ciblant initialement une catégorie d'infracteurs relativement circonscrite (trafiquants de drogue et blanchisseurs des produits de ce type de trafic), l'anti-blanchiment est maintenant promu pour identifier et sanctionner toutes les personnes physiques ou morales, des «plus faibles aux plus puissantes ", associées à des flux financiers illicites. À la faveur de ce décloisonnement de la lutte contre l'argent sale, nous aurions affaire à un instrument de régulation pouvant être mobilisé de manière indifférenciée pour gérer les illégalismes d'organisations à vocation criminelle, d'entreprises, d'hommes politiques et in fine de n'importe quel citoyen. La qualification de la fraude fiscale en infraction sous-jacente de blanchiment mettrait de facto un terme à toute forme de tolérance à l'égard de certaines transgressions commises par des acteurs économiques ou politiques bien plus légitimes socialement que des « barons de la drogue », des «trafiquants d'êtres humains » ou des «terroristes ». C'est justement cette assertion que nous prenons ici au sérieux en revenant brièvement sur sa traduction concrète, concernant les illégalismes fiscaux, avant de nous attarder sur deux cas spécifiques.

\section{La gestion différentielle des illégalismes fiscaux}

En France, l'inclusion des questions fiscales au sein du dispositif antiblanchiment ne s'est pas faite sans heurts. Effective depuis 2009, cette inclusion découle d'une directive européenne de 2005 dont la transposition nationale a ouvert un jeu d'interactions et d'intenses tractations ${ }^{98}$. Les représentants des institutions bancaires ont fustigé la direction donnée à la lutte contre l'argent sale avec l'entrée de la fraude fiscale dans ce domaine. «En France, contrairement à la plupart des autres pays européens, cela s'appliquera à tous les délits économiques et financiers et notamment à toute la fraude fiscale, à moins que la législation nationale ne soit adaptée. Sans distinction entre la criminalité organisée et une forme d'incivisme fiscal, cette disposition risque d'entraîner une paralysie de Tracfin, du fait du nombre considérable de déclarations de soupçons qui devraient affluer. Cela risque en outre d'aboutir à une mise en fiches de milliers de personnes. La FBF [Fédération bancaire fran-

97. House of Lords, European Union Committee, 19th Report of Session 2008-09: Money laundering and the financing of terrorism. Volume I : Report, London, 2009, pp. 44-45.

98. Cette section résume à grands traits des éléments développés dans Amicelle A., « Gestion différentielle des illégalismes économiques et financiers ", Champ pénal/Penal Field [En ligne], vol. 10, 2013. 
çaise] demande aux pouvoirs publics une solution pragmatique afin que les banques puissent continuer à lutter efficacement contre le blanchiment, dans un cadre juridique clarifié 99 ». Présenté comme une forme d'incivisme via cet exercice d'euphémisation à peine voilé, l'ensemble « fraude fiscale » renvoie ici à « des erreurs, pas des fautes 100 ».

Les positions exprimées par les représentants du ministère des Finances, en charge du dossier, consistaient aussi à rappeler que ce n'était pas la finalité de Tracfin d'être accaparé par les questions fiscales. Des "plans de secours » ont été échafaudés et la discussion a battu son plein lors des consultations menées sur le projet de transposition de la directive européenne. L'approche consistant à faire éclater la définition existante de la fraude fiscale a été sérieusement envisagée 101. Il s'agissait de distinguer par exemple une "fraude fiscale aggravée » d'une "fraude fiscale simple », de façon à ce que la deuxième échappe à la régulation anti-blanchiment et aux signalements à Tracfin auxquels sont astreints les acteurs bancaires en cas de soupçon d'argent sale.

La transposition n'a pas été dans le sens d'un éclatement du délit de fraude fiscale mais contrairement aux autres infractions sous-jacentes de blanchiment, le soupçon ne suffit pas dans ce cas pour transmettre une déclaration d'opération suspecte à Tracfin. Concrètement, en plus de savoir, de soupçonner ou d'avoir de bonnes raisons de soupçonner que des capitaux seraient associés à une infraction fiscale, il faut que celle-ci corresponde à un des seize critères constitutifs de la présence d'un blanchiment de fraude fiscale; une liste de critères établie par un décret spécifique du Premier ministre François Fillon. L'introduction de ces critères a été justifiée à l'aune de la complexité de la fraude fiscale afin d'aider les acteurs de première ligne à aiguiller leur vigilance à l'aide d'indicateurs. Cependant, elle a aussi pour effet de limiter les signalements pour fraude fiscale aux seules opérations répondant à au moins un des critères définis, ce qui revient mécaniquement à exclure du champ déclaratif celles pour lesquelles aucune correspondance formelle n'est relevée avec un de ces indicateurs.

In fine, la façon dont cette transposition de la directive européenne est réalisée abonde dans le sens d'une différenciation entre deux types d'illégalismes fiscaux selon une échelle de gravité aux contours imprécis. Porteuse d'ambiguité et d'incertitude, la liste des seize critères a fait l'objet d'un travail d'interprétation, auquel se sont prêtées les parties prenantes à la mise en œuvre, aussi bien les représentants de Tracfin que ceux des institutions financières et des autres secteurs concernés. Il fallait clarifier la limite floue entre, d'un côté, les illégalismes fiscaux à signaler à la cellule de renseignement finan-

99. FBF, Rapport annuel, Paris, 2007, p. 39.

100. Lascoumes P., "Des erreurs, pas des fautes », Paris, Cesdip, 1986.

101.Favarel-Garrigues G., Godefroy T. et P. Lascoumes, Les sentinelles de l'argent sale : les banques aux prises avec l'anti-blanchiment, Paris, La Découverte, 2009. 
cier, et de l'autre, ceux hors de portée du dispositif anti-blanchiment. Cette opération d'interprétation s'est cristallisée autour de l'invention-importation de la notion de «fraude fiscale grave et organisée » pour donner sens au décret ministériel. N'apparaissant ni dans ce décret, ni dans d'autres documents officiels et n'ayant aucune valeur juridique en France, cette notion s'est pourtant imposée comme un repère partagé pour qualifier les situations fiscales rencontrées afin de justifier de la nécessité ou non de les dénoncer. Cette référence est retenue par les acteurs bancaires et les fonctionnaires de Tracfin répétant à l'envi que « concernant la problématique actuelle sur la notion de la fraude fiscale que rencontrent les professionnels, Tracfin insiste sur la notion de fraude fiscale "grave et organisée” 102 ». Ainsi, l'ensemble de la fraude fiscale est théoriquement couvert par la régulation anti-blanchiment mais une partie en est concrètement préservée par le caractère sélectif du contrôle établi, des dénonciations transmises en fonction des réglementations officielles et de la manière dont elles sont interprétées.

En consacrant par la pratique une nouvelle différenciation, Tracfin et ses informateurs officialisent le fait que la fraude fiscale « ordinaire » échappe à l'application de la directive anti-blanchiment. Au-delà des routines organisationnelles et des représentations conduisant à associer anti-blanchiment et répression de la "criminalité organisée ", cette marge d'illégalismes tolérés reflète aussi la situation de Tracfin aux prises avec des impératifs pragmatiques prenant le pas sur les impératifs légaux. L’inquiétude de voir ce service submergé par un afflux de déclarations impossible à traiter a joué à plein, tout comme la volonté de ne pas entamer la relation de confiance tissée avec les milieux financiers.

Au regard de ce nouveau dégradé des illégalismes fiscaux, reste cependant à savoir où s'arrête précisément la fraude fiscale « ordinaire » et où commence la fraude fiscale " grave et organisée », pour essayer de comprendre le sort désormais réservé à la criminalité en col blanc. C’est dans ce cadre que les premières mises en examen pour blanchiment de fraude fiscale ont été prononcées ces derniers mois, dont certaines visant bien des personnalités économiques et politiques. Sans tomber dans une surinterprétation du peu de cas à notre disposition, il s'agit simplement de formuler quelques remarques exploratoires à partir de deux affaires judiciaires en cours de traitement et présentées comme le symbole de ce nouveau recours à l'anti-blanchiment contre les illégalismes fiscaux des « col-blancs », soit les dossiers Cahuzac et Lamblin.

102. Propos de Jean-Pierre Gautier (Tracfin) lors de sa communication intitulée « Le nouveau contexte d'obligations déclaratives pour les organismes financiers » à la « 9 e conférence internationale : Lutte anti-blanchiment, lutte contre la criminalité financière », 24-25 juin 2010. 
Le 2 avril 2013, 15 jours après avoir quitté ses fonctions ministérielles à la suite de l'ouverture d'une information judiciaire contre $\mathrm{X}$ pour blanchiment et fraude fiscale par le parquet de Paris, Jérôme Cahuzac reconnaît avoir détenu un compte bancaire à l'étranger, non-déclaré au fisc français ${ }^{103}$. Selon le principal intéressé secondé de son avocat, ce compte, domicilié en Suisse pendant plus de vingt ans avant d'être transféré en 2009 à Singapour, a été abondé à hauteur de 600000 euros. Ce montant est alors associé à son activité professionnelle de chirurgien esthétique et à ses services ponctuels de consultant auprès de compagnies pharmaceutiques au début des années 1990. À cet égard, les faits qui lui sont reprochés correspondent assez bien à l'expression de criminalité en col blanc définie par Sutherland, soit des actes commis par des individus de statut social élevé en rapport avec leurs activités économiques et professionnelles. Au regard de son métier principal et de la fonction de conseiller en cabinet ministériel qu'il a occupé au ministère de la Santé entre 1988 et 1991, ses honoraires de consultant lui valent d'être poursuivi pour "blanchiment de perception par un membre d'une profession médicale d'avantages procurés par une entreprise dont les services ou les produits sont pris en charge par la sécurité sociale $104 »$. Toujours en date du 2 avril, l'ancien ministre du Budget est aussi mis en examen pour blanchiment de fraude fiscale.

Ses confessions agrémentées de poursuites judiciaires ponctuent près de quatre mois de dénégations et de réactions publiques contradictoires au sujet de l'existence même du compte bancaire suisse. En effet, le premier article de presse à ce propos est publié le 4 décembre 2012 par le journaliste Fabrice Arfi dans les colonnes du quotidien en ligne Mediapart ${ }^{105}$. Si cette révélation suscite immédiatement une réaction collective, elle initie moins un «scandale » qu'une «affaire Cahuzac » dans la mesure où les sources d'indignation sont loin d'être unanimement partagées ${ }^{106}$. L'espace médiatique est grossièrement divisé en deux camps audibles, l'un accusant Cahuzac, l'autre accusant ses accusateurs en critiquant le manque d'éléments tangibles fournis pour étayer les allégations portées à l'encontre du ministre du Budget 107. Parmi ses sources, en complément d'un enregistrement sonore transmis par un tiers, le journaliste de Mediapart s'appuie notamment sur un mémoire rédigé en 2008 par un agent du fisc, aujourd'hui à la retraite, ayant alors consulté sans autorisation le dossier fiscal de Jérôme Cahuzac. Cet élément ajoute à la controverse et au durcissement des positions avec des acteurs saluant le travail obstiné d'un

103. Laurent S., « Si vous n'avez rien suivi de l'affaire Cahuzac », Le Monde, avril 2013.

104. Piel S., "Jérôme Cahuzac encourt cinq ans de prison », Le Monde, avril 2013.

105. Arfi F., « Le compte en Suisse du ministre du budget », Mediapart, décembre 2012.

106. Pour plus de précisions sur les usages sociologiques des notions d'affaire et de scandale, voir Boltanski L., Claverie E., Offenstadt N. et S. Van Damme (eds.), Affaires, scandales et grandes causes : De Socrate à Pinochet, Paris, Stock, 2007.

107. Cazi E., Chemin A., «Et Mediapart passa des critiques aux louanges », Le Monde, mars 2013. 
agent de terrain entravé par sa direction quand d'autres dénoncent une intervention abusive d'un fonctionnaire de l'État motivée par des rancœurs personnelles ${ }^{108}$. Structuré autour d'une série d'arguments normatifs opposés, un antagonisme plus ou moins radical s'installe ainsi pendant plusieurs mois entre journalistes, professionnels de la politique et citoyens, l'homme d'État endossant à tour de rôle les traits du coupable et de la victime.

Finalement, l'accumulation d'indices concordants, ayant mené à des aveux largement relayés, redéfinit sensiblement les contours d'une affaire désormais re-transformée en scandale avec un retournement unanime contre l'« ancien inspecteur en chef des impôts ». Si une sociologie de cette controverse et de ses effets reste à mener, le rappel de ces quelques éléments de contexte suffit à souligner un fait saillant pour notre analyse : la réaction sociale contre ce cas présumé de blanchiment de fraude fiscale n'a pas été initiée par les professionnels de la surveillance et du renseignement financier impliqués dans la mobilisation nationale, européenne et internationale contre l'argent sale. Les instruments de régulation anti-blanchiment ont certes été utilisés pour poursuivre en justice l'ancien membre du gouvernement mais ils n'ont aucunement servi, dans ce cas précis, à détecter les fonds et les opérations en cause. «Ce sont, une fois encore, des révélations privées relayées par les médias qui ont fait office de gendarme 109 ». C'est également le cas pour les récentes accusations de blanchiment et de fraude fiscale portées dans plusieurs pays européens contre HSBC Private Bank - une personne morale cette fois-ci - sur la base des fichiers internes dévoilés en 2008 par un ancien informaticien de la banque, Hervé Falciani.

Ce premier exemple «Cahuzac » gagne à être confronté à une autre procédure judiciaire ayant également connu un certain retentissement médiatique, à un degré bien moindre cependant, laquelle découle cette fois-ci directement d'investigations policières comprenant un volet anti-blanchiment.

\section{Plus propre que l'argent sale?}

En octobre 2012, Florence Lamblin, alors ajointe au maire du $13^{\mathrm{e}}$ arrondissement de Paris, est mise en examen pour blanchiment en bande organisée et association de malfaiteurs. Elle figure parmi les 17 individus interpellés en France dans le cadre d'une enquête criminelle ayant établi un lien original entre la plus ancienne infraction sous-jacente de blanchiment d'argent, le trafic de drogue, et la plus récente, la fraude fiscale 110 . Huit mois plus tôt, des

108. Jarrassé J., De Charrette L., «Entre Cahuzac et l'agent du fisc, un vieux conflit », Le Figaro, décembre 2012 ; Mathiot C., "L'étonnante histoire de "Columbo" et Cahuzac », Libération, décembre 2012.

109. Lascoumes P., "Contre l'argent illicite, non aux lois de panique », Mediapart, 2013, p. 1.

110. AFP, «Blanchiment d'argent de la drogue : une élue parisienne EELV parmi les 17 interpellés », AFP, octobre 2012 ; AFP, «Blanchiment: un système parfaitement rodé, des “cols blancs” peu scrupuleux », AFP, octobre 2012. 
agents de l'Office central pour la répression du trafic illicite de stupéfiants (OCRTIS) ont effectué des filatures et mis sur écoute plusieurs personnes actives dans l'importation et la revente de cannabis entre le Maroc et la capitale française. Ils ont ensuite lancé une opération conjointe avec leurs homologues de l'Office central pour la répression de la grande délinquance financière (OCRGDF) afin d'enquêter sur les pratiques de blanchiment des capitaux issus de ce trafic ${ }^{111}$. Ils découvrent alors l'existence d'un système de compensation impliquant aussi bien les trafiquants sous surveillance que des «cols blancs » selon l'expression maintes fois reprises dans les médias pour faire référence à l'élue municipale et à d'autres personnes mises en cause ${ }^{112}$.

Selon les sources policières et judiciaires relayées par voie de presse, les trafiquants suivis s'ingéniaient à recycler les produits de leur activité lucrative afin d'en dissimuler l'origine illégale pour éviter la détection et la confiscation de ces fonds. Pour ce faire, la majeure partie de l'argent liquide circulait par le biais d'intermédiaires et in fine d'un collecteur de fonds, basé à Paris, alimentant un système de compensation établi entre la France et la Suisse pour empêcher de retracer certaines opérations financières ${ }^{113}$. Hormis ce collecteur de fonds, le système aurait principalement fonctionné à l'aide de deux financiers genevois, l'un officiant comme administrateur délégué d'une société de gestion de fortune (GPF S.A.) et l'autre étant employé chez HSBC Private Bank qui rabattait les clients en mal d'évasion fiscale et avec qui GPF S.A., tout du moins son directeur, disposait de comptes bancaires, dont un à Londres 114. Dans le cadre de ses fonctions, cet administrateur délégué avait notamment affaire à des citoyens français titulaires de comptes non-déclarés. Désireux de rapatrier leurs avoirs sans attirer l'attention des autorités, l'administration fiscale ou Tracfin, ceux-ci souhaitaient y parvenir sans recourir à des virements électroniques potentiellement suspects ou au transport risqué de valises de billets entre Genève et Paris ${ }^{115}$. Ils récupéraient alors leurs capitaux à l'aide du mécanisme par lequel aurait également convergé l'argent issu du trafic de cannabis via un principe de vases communicants 116 .

Détentrice de 350000 euros sur un compte en Suisse qu'elle souhaite récupérer sans être imposée, Florence Lamblin est mise en contact avec le gérant de GPF S.A. afin de pouvoir disposer de ses fonds en toute discrétion. Le partenaire de ce dernier (le « collecteur de fonds ») remet alors le montant corres-

\section{Ibid.}

112. À titre d'exemple: «L'élue n'est pas le seul “col blanc" du dossier à avoir bénéficié de remises d'argent en espèces. Un marchand d'art, un avocat de l'avenue Montaigne, des dirigeants d'entreprise, un dentiste... ». Mallevoüe F., «Blanchiment d'argent : ce qu'on reproche à l'élue ", Le Figaro, octobre 2012.

113. Baudet P., « Blanchiment: par où passe l'argent de la drogue », Ouest France, octobre 2012;

Bordenave Y., «Sept notables, trois frères et l'argent de la drogue », Le Monde, octobre 2012. 114. Mansour F., "La chute brutale de deux financiers genevois », Le Temps, octobre 2012 ; Favre

A., «Blanchiment : la place financière surprise et préoccupée », Le Temps, octobre 2012. 
pondant à cette nouvelle cliente et ce, sans aucun mouvement transfrontalier ni lien apparent avec le compte bancaire helvétique 117. Autrement dit, Florence Lamblin a concrètement obtenu la somme demandée sous forme d'espèces préalablement collectées à Paris. Plus précisément, et c'est l'objet des poursuites engagées à son égard, elle est accusée d'avoir perçu via cette opération de l'argent directement issu du trafic de cannabis sous investigation et d'avoir ainsi contribué au blanchiment de ces capitaux ${ }^{118}$. En effet, son compte non-déclaré a ensuite été débité d'une valeur équivalente via une série de montages financiers maîtrisés par le directeur de GPF S.A., à la faveur du compte londonien chez HSBC, afin d'être reversé en bout de chaîne aux véritables propriétaires des billets de banque livrés à l'élue municipale ${ }^{119}$. Les gestionnaires du système de compensation ont facturé leurs services d'intermédiation en prélèvant une commission sur les opérations réalisées 120 .

Le dossier judiciaire porte ainsi sur un système de pratiques faisant converger deux démarches frauduleuses, «d'une part évacuer le cash des trafiquants et d'autre part permettre à des délinquants financiers de prendre possession de sommes qu'ils ont escamotées 121 ». Ces deux démarches, dont celle motivée par des considérations strictement fiscales, ont été mises à jour par une équipe d'enquêteurs spécialisés sur les flux financiers illicites. Contrairement au scandale Cahuzac, la mise en examen de Florence Lamblin et d'autres « cols blancs » parisiens résulte bien d'une investigation ayant mobilisé en amont les instruments de régulation anti-blanchiment. Néanmoins, la détection des illégalismes fiscaux n'est que le résultat collatéral d'une enquête ciblant d'abord un trafic de stupéfiants. Alors qu'il fonctionnait depuis plusieurs années au bénéfice d'évadés fiscaux, le système de compensation a finalement été repéré et ciblé parce qu'il contribuait à blanchir de l'« argent de la drogue » 122 .

«Cette affaire, d'une ampleur particulière, n'aurait sans doute jamais vu le jour sans les filatures opérées par les policiers français spécialisés dans la répression des stupéfiants. L'observation des " go fast », puis des revendeurs et des collecteurs de fonds, a conduit les enquêteurs sur la piste du compte londonien. Une commission rogatoire plus tard, le lien était fait avec la société financière de la cité de Calvin 123 ». La découverte d'un système organisé de blanchiment de fraude fiscale au profit d'acteurs économiques et politiques

117.Ibid.; Mallevoüe F., «Blanchiment d'argent : ce qu'on reproche à l'élue », op. cit.

118. Bordenave Y., "Florence Lamblin n'as pas “pensé que c'était de l'argent sale” ", Le Monde, octobre 2012.

119. Ibid. ; Frammery C., "Une affaire de blanchiment très française et très suisse ", Le Temps, octobre 2012 ; Mansour F., " Des blanchisseurs au sort presque scellé », Le Temps, janvier 2013.

120. Bordenave Y., «Florence Lamblin n'as pas “pensé que c'était de l'argent sale” », op. cit.

121. Bordenave Y., "Sept notables, trois frères et l'argent de la drogue ", Le Monde, octobre 2012.

122. Bordenave Y., «Blanchiment : le chaînon manquant entre financiers et trafiquants arrêté », Le Monde, janvier 2013.

123. Mansour F., « La chute brutale de deux financiers genevois », Le Temps, octobre 2012. 
légitimes est une conséquence inattendue de l'opération policière mise sur pied pour filer des vendeurs de cannabis et suivre leur argent à la trace. Selon un porte-parole des services d'enquête, "le rôle du collecteur central a intrigué parce qu'il livrait des billets dans des sacs plastique ou des valises à roulettes, entre 100000 et 400000 euros à des gens qui n’étaient pas dans le radar des stups. [...] Il a fallu des centaines d'heures de surveillance et d'écoutes téléphoniques pour comprendre ce "cirque" et mettre au jour ce mécanisme d'ingénierie financière sophistiquée. [...] Les clients ne voulaient certainement pas savoir d'où venait l'argent qu'ils obtenaient sur un claquement de doigts et qu'on leur remettait avec un luxe de précautions. Les sommes étaient remises en liquide dans un café ou un hôtel parisien et l'échange durait à peine 10 minutes, il n'y avait pas de comptage 124 ». Les moyens de surveillance et de renseignement financier ont été alloués pour lutter contre un trafic de stupéfiants et non pour viser des transgressions associées à des « cols blancs» 125 .

À la suite des poursuites engagées, la défense de l'ancienne élue municipale a d'ailleurs consisté à minimiser les illégalismes fiscaux en dramatisant ceux liés au trafic de cannabis. Son avocat a constamment rappelé que sa cliente n'avait pas la moindre idée de l'origine des fonds distribués en liquide et que, victime malchanceuse, elle avait peut-être pu devenir la complice involontaire d'une opération criminelle. « À l'invitation de sa banque, ma cliente a souhaité rapatrier 350000 euros provenant d'un héritage familial placé sur un compte en Suisse depuis 1920, indique Me Jérôme Boursican. Elle s'est faite conseiller par une personne de confiance qui l'a mise en contact avec une relation susceptible de traiter ce genre de transaction. Elle s'est faite avoir, pensant que les fonds récupérés étaient les siens et non pas de l'argent issu du trafic de drogue. Avait-elle conscience des activités de blanchiment de cette personne ? A l'évidence, non 126 ». Il use d'une stratégie d'euphémisation visant à réintroduire une distinction entre ce qui relève de la « légèreté 127 » et ce qui s'apparente à une véritable faute ${ }^{128}$.

Afin d'échapper au stigmate social et aux qualifications pénales, cet avocat tente donc de réinstaurer la frontière normative historiquement négociée, mais désormais abolie, entre fraude fiscale et argent sale. Contrairement aux straté-

124. Frammery C., «Une affaire de blanchiment très française et très suisse », op. cit.

125. Ce point s'applique aussi à la «Plate-forme d'identification des avoirs criminels » (PIAC) mise en place en 2005 et à l' " Agence de gestion et de recouvrement des avoirs saisis et confisqués » (AGRASC) créée en 2010. La directrice générale de l'AGRASC a tout de même indiqué le lancement d'une première analyse centrée sur la fraude fiscale. Cf. AGRASC, Rapport annuel, Paris, 2014.

126. «Blanchiment : l'embarrassante fortune de l'élue écologiste parisienne », Le Parisien, octobre 2012.

127. «Florence Lamblin, élue écologiste de Paris mise en examen, a démissionné », Le Monde, octobre 2012.

128. Sur ces stratégies d'euphémisation employées pour diluer la portée des faits et des responsabilités, voir Lascoumes P., "Élites délinquantes et résistance au stigmate », Champ pénal/Penal field [En ligne], vol 10, 2013. 
gies d'évitement légal finement analysées par Doreen McBarnet, il ne peut jouer avec les frontières du légal et de l'illégal pour s'assurer que sa cliente échappe à l'impôt tout en restant du bon côté de la légalité. Sa cliente a d'ores et déjà admis qu'elle était bien titulaire d'un compte non-déclaré et qu'elle avait sciemment essayé de récupérer son argent par des voies détournées 129. Délaissant les frontières de la légalité, la ligne de défense est alors articulée autour de la tension entre illégalismes et délinquance, ou plus précisément, dans le cas présent, entre argent propre et argent sale afin de stopper les poursuites pénales au profit d'un autre type de règlement du conflit. " Considérer le choix du mode de règlement comme un enjeu, c'est resituer les formes juridiques apparentes dans le champ des rapports de pouvoirs marquant toute interaction conflictuelle (entre particuliers, particulier-instance publique ou assimiliée) $130 »$. Tout l'enjeu revient à éviter la forme d'intervention pénale puisque les illégalismes fiscaux font partie de ces «illégalismes privilégiés » décrits par Acosta, susceptibles de connaître différentes modalités de résolution relatives à plusieurs systèmes normatifs (sanctions pénales, régularisation administrative, amendes, etc.). L'acte reproché à Florence Lamblin ne pouvant apparaître «plus blanc que la criminalité en col blanc » (whiter than white collar crime 131), son avocat a cherché à ce que son argent apparaisse plus propre que de l'argent sale. En voie de banalisation, cette rhétorique fait désormais florès sur les bancs de l'Assemblée nationale, auprès des députés signalés à la justice pour fausse déclaration de patrimoine et dissimulation d'avoirs détenus à l'étranger ${ }^{132}$. Il est à noter que ces signalements ont été faits par la nouvelle « Haute autorité pour la transparence de la vie publique », précisément chargée de contrôler les élus du Palais Bourbon. Doit-on y voir l'exception qui confirme la règle ou l'expression des premiers grondements d'une bataille sans précédent et sur plusieurs fronts contre la « déviance et délinquance des élites dirigeantes » ?

\section{Conclusion}

«Dans tout régime, les différents groupes sociaux, les différentes classes, les différentes castes ont chacun leur illégalisme 133 ». Rappelant ici l'absence d'un lien de causalité exclusif entre pauvreté et comportement transgressif, Michel Foucault s'est attaché à saisir les façons dont les illégalismes sont pratiqués et traités selon les catégories et les situations sociales. Au travers de l'attention portée à la recomposition de l'économie générale des illégalismes au sortir du XVIII siècle, il a articulé l'analyse d'un processus de transformation

129. Bordenave Y., « Florence Lamblin n'as pas “pensé que c'était de l'argent sale” », op. cit.

130. Lascoumes P., Les affaires ou l'art de l'ombre, op. cit., p. 204.

131. McBarnet D., "Whiter than White Collar Crime: Tax, Fraud Insurance and the Management of Stigma”, op. cit.

132. Cazi E., «Trois parlementaires UMP rattrapés par la justice sur leur patrimoine », Le Monde, novembre 2014.

133. Foucault M., «À propos de l'enfermement pénitentiaire ", in M. Foucault, Dits et écrits I. 1954-1975, Paris, Gallimard, 2001, p. 1303. 
historique avec une réflexion sur l'exercice du pouvoir et les rapports de domination. Depuis, plusieurs auteurs ont prolongé ce programme de recherche en prenant comme point d'entrée empirique des composantes plus ou moins variées du vaste ensemble représenté par les illégalismes économiques et financiers. Rendant compte des multiples changements opérés depuis les travaux de Foucault, ils ont malgré tout montré le maintien du sens donné à la gestion des illégalismes via des modes sans cesse renouvelés de différenciation aux effets inégalitaires; avec des déplacements, des recoupements et des superpositions entre différents couples de différenciation (illégalismes de biens/illégalismes de droits ; illégalismes privilégiés/illégalismes typiques ; illégalismes populaires/illégalismes des classes dominantes; illégalismes graves et organisés/ illégalismes ordinaires ; argent propre/argent sale).

Parmi ces chercheurs, Pierre Lascoumes a ouvert la voie en scrutant la tension stratégique entre illégalismes et délinquance à la lumière du monde des affaires. Il a rappelé comment la «criminalité d'affaires » s'est très tôt différenciée de la «criminalité ordinaire » par les modes de réaction sociale qu'elle a suscités, en insistant notamment sur son véritable privilège, à savoir la pluralité des formes juridiques susceptibles de lui être appliquée. De son côté, Alexis Spire a relevé avec d'autres que le recours aux illégalismes de droits s'est - dans une certaine mesure - diffusé à l'ensemble du corps social, quand bien même des différences significatives subsistent dans les manières d'y accéder et de procéder. Il a surtout dévoilé l'inégalité de traitement inhérente aux rapports de force engagés entre l'administration fiscale et ses administrés selon les catégories et les situations sociales. En restituant les mécanismes pratiques qui favorisent ces inégalités, il explicite ainsi le rapport à l'impôt dans la reproduction de l'ordre social. À côté de cette gestion différentielle entre illégalismes fiscaux, l'analyse du dispositif anti-blanchiment a montré la persistance d'une gestion différentielle des illégalismes fiscaux.

S'il ne résume pas à lui seul la multiplicité des instruments de régulation en place, ce dispositif se présente comme le cadre incontournable pour surveiller les détenteurs de capitaux illicites et leurs complices. À cet égard, la lutte contre le blanchiment est désormais corrélée à un dégradé d'illégalismes lucratifs comprenant des atteintes aux biens et à la propriété, mais aussi des fraudes et des «finesses de citadins» en passant par des actes de corruption et des trafics en tout genre. En tant qu'illégalismes résultant d'autres illégalismes (de biens et de droits) générant de l'argent sale, les pratiques de blanchiment (et leur contrôle) constituent une entrée majeure pour questionner l'économie actuelle des illégalismes. Mobilisant des technologies de surveillance, impliquant des agents de différents organismes étatiques mais aussi des street level bureaucrats d'une dizaine de secteurs économiques, et comportant une dimension transnationale, la régulation anti-blanchiment est devenue l'instrument de gestion de l'argent illicite dans toutes ses manifestations. Comment 
les acteurs de ce dispositif différencient, catégorisent, hiérarchisent et gèrentt-il quotidiennement les flux financiers mis à l'index ? Alors que le droit pénal est le code de lecture retenu pour punir les actes de blanchiment, quelles sont les rhétoriques de défense employées et les modes de résolution privilégiés selon le type d'illégalismes et selon les personnes morales et/ou physiques concernées ? Dans quelle mesure cette lutte contre l'argent sale participe-t-elle au changement ou à la protection de l'ordre économique et financier existant ? Autant de questions qui aiguillent notre enquête de terrain en cours, au sein d'un département universitaire où Michel Foucault a aussi été convoqué pour défendre l'idée que la criminologie pouvait être " autre chose qu'une science de corps de garde, où l'on rit gras et on cogne dur quand on rencontre des mots de plus de trois syllabes ${ }^{134} »$. 